\title{
On the automorphism group of foliations with geometric transverse structures
}

\author{
Laurent Meersseman ${ }^{1} \cdot$ Marcel Nicolau $^{2} \cdot$ Javier Ribón $^{3}$
}

Received: 25 March 2021 / Accepted: 26 November 2021 / Published online: 22 January 2022

(c) The Author(s) 2022

\begin{abstract}
We study the structure of some groups of diffeomorphisms preserving a foliation. We give an example of a $C^{\infty}$ foliation whose diffeomorphism group has not a natural structure of Lie group. On the positive side, we prove that the automorphism group of a transversely holomorphic foliation or a Riemannian foliation is a strong ILH Lie group in the sense of Omori. We also investigate the relationship of the previous considerations with deformation problems in foliation theory. We show that the existence of a local moduli space for a given foliation imposes strong conditions on its automorphism group. They are not fulfilled in many cases, in particular they are not fulfilled by the foliation mentioned above.
\end{abstract}

Mathematics Subject Classification 58D05 $\cdot$ 53C12 $\cdot$ 22E65 $\cdot 58 \mathrm{D} 27 \cdot 22 \mathrm{~F} 50$

\section{Introduction}

Let $M$ be a closed smooth manifold. We denote by $\mathcal{D}=\mathcal{D}(M)$ the group of all $C^{\infty}$. diffeomorphisms of $M$ endowed with the $C^{\infty}$ topology. It is a metrizable topological group. In [15], Leslie proved that $\mathcal{D}$ is a Fréchet Lie group; namely, a group with a structure of infinite dimensional manifold modeled on a Fréchet space such that the group operations are smooth. Fréchet manifolds are difficult to deal with as many of the fundamental results of calculus,

This work was partially supported by the grants MTM2015-66165-P, MTM2016-77642-C2-1-P and PGC2018-095998-B-I00 from the Ministerio de Economia y Competitividad of Spain and by Conselho Nacional de Desenvolvimento Científico e Tecnológico-CNPq, Proc. 308838/2019-0.

Marcel Nicolau

nicolau@mat.uab.cat

Laurent Meersseman

laurent.meersseman@univ-angers.fr

Javier Ribón

jribon@id.uff.br

1 Laurent Meersseman, Univ Angers, CNRS, Larema, SFR Mathstic, 49000 Angers, France

2 Departament de Matemàtiques, Universitat Autònoma de Barcelona, 08193 Cerdanyola, Spain

3 Instituto de Matemática e Estatística, Universidade Federal Fluminense, Niterói, Rio de Janeiro, Brazil 
like implicit function theorem or Frobenius theorem, are not valid in that category. However, Palais proved that $\mathcal{D}$ has a richer structure: it is a ILH Lie group, that is a topological group that is the inverse limit of Hilbert manifolds $\mathcal{D}^{k}$ each of which is a topological group [22,25]. Later on, Omori introduced the notion of strong ILH Lie group, whose precise definition is recalled in 2.1. The interest of this stronger notion is that it allows to formulate an implicit function theorem and a Frobenius theorem in that setting (cf. [23]). In addition to demonstrate those results, Omori used them to show that $\mathcal{D}$ is a strong ILH Lie group.

It is natural to ask for similar results for groups of diffeomorphisms preserving a geometric structure. In [6], Ebin and Marsden proved the existence of a structure of a ILH Lie group on $\mathcal{D}_{\eta}$ and $\mathcal{D}_{\omega}$, the subgroups of smooth diffeomorphisms preserving a volume element $\eta$ or a symplectic form $\omega$ on $M$ respectively; and Omori showed that some subgroups of $\mathcal{D}$, including $\mathcal{D}_{\eta}$ and $\mathcal{D}_{\omega}$, are strong ILH Lie groups (cf. [23,24]).

In this paper, we are interested in the structure of groups of diffeomorphisms preserving a foliation, and preserving a foliation with some additional geometric transverse structure. Our main motivation comes from deformation problems. We want to understand the relationship between the existence of a nice local moduli space for a certain type of foliations and the structure of their automorphism groups.

It turns out that the structure of the automorphism group of a smooth foliation is much more complicated than the structure of the subgroups of $\mathcal{D}$ mentioned above. Indeed, Omori's techniques are well-suited for groups of transformations of compact manifolds generating primitive infinite pseudo-groups, which exclude explicitly groups preserving a foliation. We give in this paper a negative result but also two positive ones.

Let us assume first that $\mathcal{F}$ is a smooth foliation on $M$. In [16], Leslie asserted that the group of foliation preserving diffeomorphisms $\mathcal{D}_{\mathcal{F}}$ is a Fréchet Lie group. Unfortunately there was a gap in his demonstration, which remains only valid for foliations with a transverse invariant connection. Indeed, for certain foliations the group $\mathcal{D}_{\mathcal{F}}$ has not a natural structure of Lie group. This fact seems to be folklore although, as far as we know, there is no published example. In part 2, we construct and discuss in detail such a foliation.

Let us now consider a type of geometric structure $\Xi$ having the property that if $\epsilon$ is a structure of type $\Xi$ on a compact manifold $M$ then the automorphism group $\operatorname{Aut}(M, \epsilon)$ is a Lie group in a natural way. Assume that the foliation $\mathcal{F}$ is endowed with a geometric transverse structure of type $\Xi$. Because of the previous negative result, there is no reason for the automorphism group $\operatorname{Aut}(\mathcal{F})$ (that is the group of the diffeomorphisms of $M$ which preserve the foliation as well as its geometric transverse structure) to be a strong ILH Lie group. We show this is nevertheless true in the following two cases: $\mathcal{F}$ is a transversely holomorphic foliation (part 4) or a Riemannian foliation (part 5). In both cases, we also show the existence of a foliated structure on $\operatorname{Aut}(\mathcal{F})$ which is transversely modeled on a finite-dimensional Lie group.

Part 3 investigates the impact of the previous results on deformation problems in foliation theory. We want to understand the local deformations/moduli space of smooth foliations possibly with a geometric transverse structure. Of course we would like to understand which of these spaces have a natural structure of manifold, or analytic space. In contrast with the theory of deformations of complex structures on a compact manifold, there are no general results for deformations of foliated structures except for the transversely holomorphic case [7]. We introduce the notion of Kuranishi property (cf. Definition 3.1), which is inspired in the deformation theory of complex structures. A foliation satisfying that property has a nice local moduli space. But we show that this property imposes strong constraints on the automorphism group of the foliation. In particular, it must be locally path-connected, so the example constructed in part 2 does not fulfill that property. We then study more thoroughly 
the case of foliations defined by suspension of a diffeomorphism of the circle (its holonomy map). If such a foliation fulfills the Kuranishi property then its holonomy map fulfills an analogous property imposing strong constraints on its centralizer. Especially, we show that a diffeomorphism of the circle with the Kuranishi property cannot have an irrational rotation number. All these facts make clear that the Kuranishi property is only fulfilled by quite special foliations.

\section{Groups of diffeomorphisms preserving a foliation}

Throughout this article $M$ will be a fixed closed smooth manifold. Let $\mathfrak{X}=\mathfrak{X}(M)$ be the Lie algebra of smooth (of class $C^{\infty}$ ) vector fields on $M$. Endowed with the $C^{\infty}$-topology, $\mathfrak{X}$ is a separable Fréchet space. We denote by $\mathcal{D}=\mathcal{D}(M)$ the group of smooth (of class $C^{\infty}$ ) diffeomorphisms of $M$ endowed with the $C^{\infty}$ topology. It is a topological group, which is metrizable and separable; so it has second countable topology.

We recall that a Fréchet manifold is a Hausdorff topological space with an atlas of coordinate charts taking values in Fréchet spaces in such a way that the coordinate changes are smooth maps (i.e. they admit continuous Fréchet derivatives of all orders). A Fréchet Lie group is a Fréchet manifold endowed with structure of group such that multiplication and inverse maps are smooth. In [15], it was proved by Leslie that $\mathcal{D}$ is a Fréchet Lie group with Lie algebra $\mathfrak{X}$. In fact, $\mathcal{D}$ is endowed with a richer structure: it is the inverse limit of Hilbert manifolds $\mathcal{D}^{k}$, each of them being a topological group [6,22]. Moreover $\mathcal{D}$ has in fact the structure of a strong ILH-Lie group [23], a notion introduced by Omori that can be defined as follows.

Recall that a Sobolev chain is a system $\left\{\mathbb{E}, E^{k}, k \in \mathbb{N}\right\}$ where $E^{k}$ are Hilbert spaces, with $E^{k+1}$ linearly and densely embedded in $E^{k}$, and $\mathbb{E}=\bigcap E^{k}$ has the inverse limit topology.

Definition 2.1 A topological group $G$ is said to be a strong ILH-Lie group modeled on a Sobolev chain $\left\{\mathbb{E}, E^{k}, k \in \mathbb{N}\right\}$ if there is a family $\left\{G^{k}, k \in \mathbb{N}\right\}$ fulfilling

(1) $G^{k}$ is a smooth Hilbert manifold, modeled on $E^{k}$, and a topological group; moreover $G^{k+1}$ is embedded as a dense subgroup of $G^{k}$ with inclusion of class $C^{\infty}$.

(2) $G=\bigcap G^{k}$ has the inverse limit topology and group structure.

(3) Right translations $R_{g}: G^{k} \rightarrow G^{k}$ are $C^{\infty}$.

(4) Multiplication and inversion extend to $C^{m}$ mappings $G^{k+m} \times G^{k} \rightarrow G^{k}$ and $G^{k+m} \rightarrow G^{k}$ respectively.

(5) Let $\mathfrak{g}^{k}$ be the tangent space of $G^{k}$ at the neutral element $e$ and let $T G^{k}$ be the tangent bundle of $G^{k}$. The mapping $d R: \mathfrak{g}^{k+m} \times G^{k} \rightarrow T G^{k}$, defined by $d R(u, g)=d R_{g} u$, is of class $C^{m}$.

(6) There is a local chart $\zeta: \tilde{U} \subset \mathfrak{g}^{1} \rightarrow G^{1}$, with $\zeta(0)=e$ whose restriction to $\tilde{U} \cap \mathfrak{g}^{k}$ gives a local chart of $G^{k}$ for all $k$.

Remarks 2.2 (a) The strong ILH-Lie group $\mathcal{D}$ is modeled on the Sobolev chain $\left\{\mathfrak{X}, \mathfrak{X}^{k}, k \in\right.$ $\mathbb{N}$ \}, where $\mathfrak{X}^{k}$ are the Sobolev completions of $\mathfrak{X}$. In that case $G^{k}=\mathcal{D}^{k}$ are the Sobolev completions of $\mathcal{D}$ and there are natural identifications $\mathfrak{g} \equiv \mathfrak{X}$ and $\mathfrak{g}^{k} \equiv \mathfrak{X}^{k}$ (cf. [23], Theorem 2.1.5). As chart $\zeta$ satisfying condition 6) in the above definition, one can take the following mapping. Fix a Riemannian metric on $M$. Given $\xi \in \mathfrak{X}$, let $\zeta(\xi)$ be the diffeomorphism whose value at $x \in M$ is $\gamma(1)$ for $\gamma$ the geodesic starting at $x$ with initial velocity $\xi(x)$. 
(b) Weaker definitions of ILH-Lie groups were considered in articles prior to [23], in particular in $[6,22]$. In the present paper we will only consider ILH-Lie groups in the strong sense given by the above definition.

(c) This strong definition ensures, by means of the last condition, that $G$ is endowed with a structure of Fréchet Lie group. The space $\mathfrak{g}=\cap \mathfrak{g}^{k}$ with the inverse limit topology is a Fréchet space. Moreover, it is naturally endowed with a Lie algebra structure (cf. [23], I.3). We call $\mathfrak{g}$ the Lie algebra of $G$.

Suppose that $H$ is a subgroup of a strong ILH-Lie group $G$ and let $\mathfrak{B}$ be a basis of neighborhoods of $e$ in $G$. For each $U \in \mathfrak{B}$, let $U_{0}(H)$ be the set of points $x \in U \cap H$ such that $x$ and $e$ can be joined by a piecewise smooth curve $c(t)$ in $U \cap H$. Here, piecewise smooth means that the mapping $c:[0,1] \rightarrow G^{k}$ is piecewise of class $C^{1}$ for each $k$. Then the family $\mathfrak{B}_{0}(H)=\left\{U_{0}(H) \mid U \in \mathfrak{B}\right\}$ satisfies the axioms of neighborhoods of $e$ of topological groups. As in [23], this topology will be called the LPSAC-topology of $H$, where LPSAC stands for "linear piecewise smooth arc-connected". In general the LPSAC-topology does not coincide with the induced topology.

Definition 2.3 Let $G$ be a strong ILH-Lie group and let $\mathfrak{g}^{k}$ be the tangent space of $G^{k}$ at $e$. A subgroup $H$ of $G$ is called a strong ILH-Lie subgroup of $G$ if there is a splitting $\mathfrak{g}=\mathfrak{h} \oplus \mathfrak{f}$ such that the following two conditions are fulfilled:

(i) It induces splittings $\mathfrak{g}^{k}=\mathfrak{h}^{k} \oplus \mathfrak{f}^{k}$ for each $k$, where $\mathfrak{h}^{k}$ and $\mathfrak{f}^{k}$ are, respectively, the closures of $\mathfrak{h}$ and $\mathfrak{f}$ in $\mathfrak{g}^{k}$.

(ii) There is a local chart $\zeta: \tilde{U} \subset \mathfrak{g}^{1} \rightarrow G^{1}$ fulfilling condition 6) in Definition 2.1 such that $\zeta(\tilde{U} \cap \mathfrak{h})=U_{0}(H)$, where $U=\zeta(\tilde{U} \cap \mathfrak{g})$

Remark 2.4 Clearly, a strong ILH-Lie subgroup $H$ of a strong ILH-Lie group $G$ is itself a strong ILH-Lie group under the LPSAC-topology.

In [23], Omori was able to state some of the classical theorems of analysis, like the implicit function theorem or Frobenius theorem, in the setting of strong ILH-Lie groups. The following is a special case of Frobenius theorem that will be used later (cf. [23], Theorem 7.1.1 and Corollary 7.1.2).

Theorem 2.5 (Omori) Let $E$ and $F$ be Riemannian vector bundles over $M$ and let $A: \Gamma(T M) \rightarrow \Gamma(E)$ and $B: \Gamma(E) \rightarrow \Gamma(F)$ be differential operators of order $r \geq 0$ with smooth coefficients. Assume that the following conditions are fulfilled:

(i) $B A=0$.

(ii) $A A^{*}+B^{*} B$ is an elliptic differential operator, where $A^{*}, B^{*}$ are formal adjoints of $A, B$ respectively.

(iii) $\mathfrak{h}=\operatorname{ker} A$ is a Lie subalgebra of $\mathfrak{X}=\Gamma(T M)$.

Then there is a strong ILH-Lie subgroup $H$ of $\mathcal{D}$ whose Lie algebra is $\mathfrak{h}$. Moreover, $H$ is an integral submanifold of the distribution $\mathcal{H}$ in $T \mathcal{D}$ given by right translation of $\mathfrak{h}$, i.e. $\mathcal{H}=\left\{d R_{f} \mathfrak{h} \mid f \in \mathcal{D}\right\}$.

Here, the notation $\Gamma(E)$ stands for the space of smooth sections of class $C^{\infty}$ of a given vector bundle $E$.

As already remarked, the group $H$ in the above theorem is a strong ILH-Lie group with the LPSAC-topology. However that topology on $H$ can be stronger than the topology induced by $\mathcal{D}$, even if $H$ is closed in $\mathcal{D}$. Therefore the above theorem is not sufficient by itself to assure 
that $H$, endowed with the induced topology, is a strong ILH-Lie group, or even a Fréchet Lie group. The following Proposition gives sufficient conditions for the above two topologies to coincide. (cf. [23], VII.1).

Proposition 2.6 (Omori) Let $H$ be a strong ILH-Lie subgroup of $\mathcal{D}$ and assume that the following conditions are also fulfilled

(a) $H$ is closed in $\mathcal{D}$,

(b) the LPSAC-topology of $H$ is second countable.

Then the topology on $H$ induced by $\mathcal{D}$ coincides with the LPSAC-topology.

From now on we assume that $M$ is endowed with a smooth foliation $\mathcal{F}$ of dimension $p$ and codimension $m$. We denote by $T \mathcal{F}$ its tangent bundle and by $\nu \mathcal{F}=T M / T \mathcal{F}$ the normal bundle of $\mathcal{F}$. The foliation $\mathcal{F}$ decomposes the manifold into the disjoint union of $p$-dimensional connected submanifolds that are called the leaves of the foliation.

An atlas adapted to $\mathcal{F}$ is a smooth atlas $\left\{\left(U_{i}, \varphi_{i}\right)\right\}$ of $M$, with $\varphi_{i}: U_{i} \rightarrow \mathbb{R}^{p} \times \mathbb{R}^{m}$ homeomorphisms, such that the leaves $L$ of $\mathcal{F}$ are defined on $U_{i}$ by the level sets $\phi_{i}=$ constant of the submersions $\phi_{i}=\pi_{2} \circ \varphi_{i}$. Here $\pi_{2}: \mathbb{R}^{p} \times \mathbb{R}^{m} \rightarrow \mathbb{R}^{m}$ denotes the projection onto the second factor. In that case there is a cocycle $\left\{\gamma_{i j}\right\}$ of local smooth transformations of $\mathbb{R}^{m}$ such that

$$
\phi_{j}=\gamma_{j i} \circ \phi_{i}
$$

The subsets $\phi_{i}=$ constant are called the slices of $\mathcal{F}$ in $U_{i}$.

We consider now the following subgroups of $\mathcal{D}$ of foliation preserving diffeomorphisms

$$
\begin{aligned}
& \mathcal{D}_{\mathcal{F}}=\left\{f \in \mathcal{D} \mid f^{*} \mathcal{F}=\mathcal{F}\right\}, \\
& \mathcal{D}_{\mathcal{L}}=\left\{f \in \mathcal{D}_{\mathcal{F}} \mid f(L)=L, \quad \text { for each leaf } L\right\} .
\end{aligned}
$$

We denote by $\mathfrak{X}_{\mathcal{F}}$ the Lie algebra of foliated vector fields (i.e. of vector fields whose flows preserve the foliation) and by $\mathfrak{X}_{\mathcal{L}}$ the Lie algebra of vector fields that are tangent to $\mathcal{F}$ (i.e. vector fields that are smooth sections of $T \mathcal{F}$ ). Notice that the flows associated to vector fields of $\mathfrak{X}_{\mathcal{F}}$ and $\mathfrak{X}_{\mathcal{L}}$ define one-parameter subgroups of $\mathcal{D}_{\mathcal{F}}$ and $\mathcal{D}_{\mathcal{L}}$ respectively. The following result was proved by Omori in [23]. We include a sketch of proof for later use.

Proposition 2.7 (Omori) The group $\mathcal{D}_{\mathcal{L}}$ is a strong ILH-Lie subgroup of $\mathcal{D}$ with Lie algebra $\mathfrak{X}_{\mathcal{L}}=\Gamma(T \mathcal{F})$.

Proof Let $\pi: \Gamma(T M) \rightarrow \Gamma(\nu \mathcal{F})$ be the linear mapping induced by the natural projection $T M \rightarrow \nu \mathcal{F}$. We can regard $\pi$ as differential operator of order 0 and its adjoint operator $\pi^{*}$, constructed using Riemannian metrics on $T M$ and $\nu \mathcal{F}$, is also a differential operator of order 0 such that $\pi \pi^{*}$ is elliptic (i.e. an isomorphism). Theorem 2.5 implies now that there is a strong ILH-Lie subgroup $\mathcal{D}_{\mathcal{L}}^{\prime}$ of $\mathcal{D}$ with Lie algebra $\mathfrak{X}_{\mathcal{L}}$. Let $\mathcal{D}_{\mathcal{L}}^{k}$ and $\mathcal{D}_{\mathcal{L}}^{\prime k}$ be the Sobolev completions of $\mathcal{D}_{\mathcal{L}}$ and $\mathcal{D}_{\mathcal{L}}^{\prime}$ respectively. Clearly $\mathcal{D}_{\mathcal{L}}^{\prime} \subset \mathcal{D}_{\mathcal{L}}$ and $\mathcal{D}_{\mathcal{L}}^{\prime k} \subset \mathcal{D}_{\mathcal{L}}^{\widetilde{k}}$. Since $\mathcal{D}_{\mathcal{L}}^{\prime}$ is obtained by the Frobenius theorem, if a piecewise $C^{1}$-curve $c(t)$ satisfies $c(0)=e$ and $c(t) \in \mathcal{D}_{\mathcal{L}}^{k}$, then $c(t) \in \mathcal{D}_{\mathcal{L}}^{\prime k}$. Using the exponential mapping with respect to a connection under which $\mathcal{F}$ is parallel, one can see that $\mathcal{D}_{\mathcal{L}}^{k}$ has a structure of Hilbert manifold, hence it is LPSAC. This implies that $\mathcal{D}_{\mathcal{L}}^{\prime k}=\mathcal{D}_{\mathcal{L}, 0}^{k}$ (the connected component of $\mathcal{D}_{\mathcal{L}}^{k}$ containing $e$ ) and therefore that $\mathcal{D}_{\mathcal{L}}^{\prime}$ is the connected component of $\mathcal{D}_{\mathcal{L}}$ containing the identity.

Remark 2.8 It follows from the above Proposition that $\mathcal{D}_{\mathcal{L}}$ is a strong ILH-Lie group with the LPSAC-topology and, in particular, it is endowed with a structure of Fréchet Lie group. In general, however, $\mathcal{D}_{\mathcal{L}}$ is not closed in $\mathcal{D}$. This is the case for instance if $\mathcal{F}$ is a linear foliation on the 2-torus $T^{2}$ with irrational slope. 
In [16], Leslie asserted that the group $\mathcal{D}_{\mathcal{F}}$ is a Fréchet Lie group. Unfortunately there was an error in his demonstration, which remains valid only for Riemann's foliations or, more generally, for foliations with a transverse invariant connection. In fact, the group $\mathcal{D}_{\mathcal{F}}$ may not be a Lie group for any sensible choice of the underlying topology of $\mathcal{D}_{\mathcal{F}}$. That fact seems to be folklore although, as far as we know, there is no published example. We exhibit here a concrete one. More precisely we prove

Theorem 2.9 There is a foliation $\mathcal{F}$ on the 2-torus $T^{2}$ such that $\mathcal{D}_{\mathcal{F}}$ cannot be endowed with a structure of a topological group fulfilling

(i) The topology of $\mathcal{D}_{\mathcal{F}}$ is finer than the $C^{0}$-topology.

(ii) $\mathcal{D}_{\mathcal{F}}$ has second countable topology.

(iii) $\mathcal{D}_{\mathcal{F}}$ is locally path-connected.

We dedicate the rest of the section to prove that statement.

Assume that an orientation preserving diffeomorphism $h \in \mathcal{D}\left(S^{1}\right)$ has been given. Let $\tau: \mathbb{R} \rightarrow \mathbb{R}$ be the translation $\tau(t)=t+1$ and let $\tilde{h}: \mathbb{R} \times S^{1} \rightarrow \mathbb{R} \times S^{1}$ denote the map defined by

$$
\tilde{h}(t, x)=\left(\tau(t), h^{-1}(x)\right)=\left(t+1, h^{-1}(x)\right) .
$$

Since $h$ is isotopic to the identity, the quotient manifold $M=\mathbb{R} \times S^{1} /\langle\tilde{h}\rangle$ is the 2-torus $T^{2}$. The foliation $\tilde{\mathcal{F}}$ of $\mathbb{R} \times S^{1}$ whose leaves are $\mathbb{R} \times\{x\}$ is preserved by $\tilde{h}$. Hence, $\tilde{\mathcal{F}}$ induces a well defined foliation $\mathcal{F}$ on $M$, which is transverse to the fibres of the natural projection

$$
\pi: \mathbb{R} \times S^{1} /\langle\tilde{h}\rangle \longrightarrow S^{1}=\mathbb{R} /\langle\tau\rangle .
$$

We say that $\mathcal{F}$ is the foliation on $T^{2}$ obtained by suspension of $h$.

We consider in $\mathcal{D}_{\mathcal{F}}$ and $\mathcal{D}\left(S^{1}\right)$ the topology of uniform convergence. We fix a circle $C=\pi^{-1}\left(t_{0}\right)$ inside the 2-torus $T^{2}$. Consider a neighborhood $U:=\left(t_{0}-\epsilon, t_{0}+\epsilon\right) \times S^{1}$ of $C$ in the torus for $\epsilon>0$ sufficiently small. Each element $f \in \mathcal{D}_{\mathcal{F}}$ close enough to the identity map Id sends $C$ into $U$. Moreover, in the adapted coordinates $(t, x)$, it is written

$$
f(t, x)=\left(f_{1}(t, x), f_{2}(x)\right) .
$$

Therefore, there is a neighborhood $\mathcal{W}$ of $\operatorname{Id}$ in $\mathcal{D}_{\mathcal{F}}$ such that the map

$$
\begin{aligned}
P: \mathcal{W} & \rightarrow \mathcal{D}\left(S^{1}\right) \\
f & \mapsto f_{2}
\end{aligned}
$$

is well-defined. Notice that $P$ is a continuous map; it is also a morphism of local groups.

Lemma 2.10 The map $P$ sends $\mathcal{W}$ into the centralizer $Z^{\infty}(h)$ of $h$ in $\mathcal{D}\left(S^{1}\right)$. Moreover, $P$ has a continuous right-inverse map $\sigma$ defined on a neighborhood $\mathcal{V}$ of the identity in $Z^{\infty}(h)$ in the $C^{0}$-topology.

Remark 2.11 It follows from the above statement that the image of the map $P$ contains a neighborhood of the identity in $Z^{\infty}(h)$.

Proof The first assertion follows from an easy computation. Given an element $g_{1} \in Z^{\infty}(h)$ close enough to the identity, the map $\tilde{g}: \mathbb{R} \times S^{1} \rightarrow \mathbb{R} \times S^{1}$ defined by $\tilde{g}(t, x)=\left(t, g_{1}(x)\right)$ commutes with $\tilde{h}$ and preserves the foliation $\mathcal{F}$. Hence, it induces an element $g \in \mathcal{D}_{\mathcal{F}}$ which is close to the identity. The map $g_{1} \mapsto \sigma\left(g_{1}\right)=g$ is well-defined on a neighborhood $\mathcal{V}$ of the identity in $Z^{\infty}(h)$. Clearly, the map $\sigma$ is continuous and fulfills $P \circ \sigma=$ Id. 
Denote by $\mathcal{D}^{r}\left(S^{1}\right)$ the group of diffeomorphisms of $S^{1}$ of class $r$, where $0 \leq r \leq \infty$, endowed with the $C^{r}$-topology. For a given $h \in \mathcal{D}\left(S^{1}\right)=\mathcal{D}^{\infty}\left(S^{1}\right)$, we denote by $Z^{r}(h)$ the centralizer of $h$ in $\mathcal{D}^{r}\left(S^{1}\right)$, and we denote by $Z_{0}^{r}(h)$ the closure in $\mathcal{D}^{r}\left(S^{1}\right)$ of the group $\langle h\rangle$ generated by $h$. Notice that $Z_{0}^{r}(h) \subset Z^{r}(h)$.

Let

$$
\rho: \mathcal{D}^{0}\left(S^{1}\right) \rightarrow S^{1}
$$

be the map that associates to each element $h \in \mathcal{D}^{0}\left(S^{1}\right)$ its rotation number $\rho(h)$. We assume that $h \in \mathcal{D}^{\infty}\left(S^{1}\right)$ has been given and that its rotation number $\alpha=\rho(h)$ is irrational. Because of Denjoy's theorem, there is a homeomorphism $\varphi$ of $S^{1}$ conjugating $h$ to the rotation $R_{\alpha}$, i.e. $\varphi \circ h \circ \varphi^{-1}=R_{\alpha}$. Since $\alpha$ is irrational, the centralizer of $R_{\alpha}$ in $\mathcal{D}^{0}\left(S^{1}\right)$ is the group of rotations. Hence the centralizer $Z^{0}(h)$ is the set $\left\{\varphi^{-1} \circ R_{\beta} \circ \varphi\right\}$ with $\beta \in S^{1}$. The natural inclusion

$$
\iota: Z^{\infty}(h) \hookrightarrow Z^{0}(h) \cong S^{1}
$$

is a continuous group morphism mapping $Z^{\infty}(h)$ onto a subgroup of $S^{1}$ (cf. [29], p. 185) for both topologies that we considered in $Z^{\infty}(h)$, namely the $C^{0}$ and $C^{\infty}$ topologies. Note that the map $\iota$ is just the restriction of $\rho$ to $Z^{\infty}(h)$. Notice also that the identification of $Z^{\infty}(h)$ with a subgroup of $S^{1}$ given by $\iota$ is algebraic but not topological.

We can consider a distance $d_{\infty}$ defining the Whitney topology of $\mathcal{D}^{\infty}\left(S^{1}\right)$ since such a space is metrizable. We set

$$
K_{\infty}(f)=\inf _{n \geq 1} d_{\infty}\left(f^{n}, \text { Id }\right)
$$

Lemma $2.12 K_{\infty}^{-1}(0)=A \cup B$ where $A$ is the subset of $\mathcal{D}^{\infty}\left(S^{1}\right)$ of elements $f$ such that $Z_{0}^{\infty}(f)$ has the cardinality of the continuum and $B$ is the subset of $\mathcal{D}^{\infty}\left(S^{1}\right)$ of elements of finite order.

Proof Let $f$ be an element of $K_{\infty}^{-1}(0) \backslash B$. Each neighborhood of the identity contains non trivial elements of $\langle f\rangle$ and therefore of its closure $Z_{0}^{\infty}(f)$ with respect to the $C^{\infty}$-topology. Hence the group $Z_{0}^{\infty}(f)$ is closed and non-discrete. As $Z_{0}^{\infty}(f)$ is perfect, it contains a Cantor set and its cardinality is bigger or equal to the cardinality of the continuum $2^{\aleph_{0}}$. In fact $Z_{0}^{\infty}(f)$ is separable, as it is a space of smooth functions on a compact manifold, and therefore its cardinality is that of the continuum. This shows that $K_{\infty}^{-1}(0) \subset B \cup A$.

Clearly $B$ is a subset of $K_{\infty}^{-1}(0)$. Let us show that each element $f \in A$ belongs to $K_{\infty}^{-1}(0)$. The group $Z_{0}^{\infty}(f)$ cannot be discrete. Otherwise it would be countable, since it is separable, getting a contradiction. We deduce that there is a sequence $\left(f^{n_{k}}\right)_{k \geq 1}$ of pairwise different elements that converges. Notice that $K_{\infty}^{-1}(0)$ is closed under inversion. Hence we can assume that $n_{k}>0$. Moreover we can also assume that the sequence $n_{k}$ is strictly increasing. We choose a sequence $m_{k}=n_{q(k)}-n_{k}$ such that $q(k)>k$ for each $k \in \mathbb{N}$ and such that $\left(m_{k}\right)_{k \geq 1}$ is strictly increasing. Then $\left(f^{m_{k}}\right)_{k \geq 1}$ is a sequence of non-trivial elements converging to the identity and therefore $f$ belongs to $K_{\infty}^{-1}(0)$.

Given an irrational number $\alpha \in S^{1}-\mathbb{Q} / \mathbb{Z}$, set

$$
Z_{0}^{\infty}(\alpha)=\bigcap_{\rho(f)=\alpha} Z_{0}^{\infty}(f)
$$

where the groups $Z_{0}^{\infty}(f)$ are thought as subsets of $S^{1}$. The following result was proved by Yoccoz (cf. [29], Théorème 3.5, p. 190) 
Theorem 2.13 (Yoccoz) There is a residual set $\mathcal{C}$ of numbers $\alpha \in S^{1}-\mathbb{Q} / \mathbb{Z}$ such that $Z_{0}^{\infty}(\alpha)$ has the cardinality of the continuum.

Corollary 2.14 There is an element $h_{0} \in \mathcal{D}^{\infty}\left(S^{1}\right)$ whose rotation number $\alpha$ is Liouville and such that $Z^{\infty}\left(h_{0}\right)$ has the cardinality of the continuum. Moreover, each continuous path $\gamma:[0,1] \rightarrow Z^{\infty}\left(h_{0}\right)$ is constant where we consider the $C^{0}$ topology in $Z^{\infty}\left(h_{0}\right)$.

Remark 2.15 We recall that for each Liouville number $\alpha$ there is a diffeomorphism of $S^{1}$ of rotation number $\alpha$ that is not $C^{\infty}$-conjugated to a rotation. This result was proved by Herman in [12, Chapter XI]. Yoccoz proved that, on the contrary, all diffeomorphisms with a diophantine rotation number are $C^{\infty}$-conjugated to a rotation [28].

Proof Since the set of Liouville numbers is residual and the intersection of two residual sets is residual, we can find an element $h_{0} \in \mathcal{D}^{\infty}\left(S^{1}\right)$ whose rotation number $\alpha=\rho\left(h_{0}\right)$ is Liouville and belongs to the set $\mathcal{C}$ in the above theorem, and such that $h_{0}$ is not $C^{\infty}$-conjugated to the rotation $R_{\alpha}$. Since $\alpha \in \mathcal{C}$ we deduce that there are non trivial elements of $Z^{\infty}\left(h_{0}\right)$ arbitrarily close to the identity in the $C^{\infty}$-topology. Let $\gamma:[0,1] \rightarrow Z^{\infty}\left(h_{0}\right)$ be a continuous path. The path is constant if and only if the composition

$$
\rho \circ \gamma:[0,1] \rightarrow Z^{\infty}\left(h_{0}\right) \hookrightarrow Z^{0}\left(h_{0}\right) \cong S^{1}
$$

is constant. If this were not the case the image $(\rho \circ \gamma)([0,1])$ would contain diophantine rotation numbers. In particular there would be an element $f$ in $Z^{\infty}\left(h_{0}\right)$ which is $C^{\infty}$ conjugated to a rotation, but then the same map would conjugate $h_{0}$ to $R_{\alpha}$ leading to a contradiction.

Proof of Theorem 2.9 Let $h_{0}$ be the element of $\mathcal{D}^{\infty}\left(S^{1}\right)$ given by the above corollary and let $\mathcal{F}$ be the foliation on $T^{2}$ obtained by suspension of $h_{0}$. Suppose that $\mathcal{D}_{\mathcal{F}}$ is endowed with a structure of locally path-connected topological group such that the topology $\mathcal{T}$ of $\mathcal{D}_{\mathcal{F}}$ is second countable and finer than the $C^{0}$-topology. There exists a neighborhood $\mathcal{W}_{0}$ of the identity in $\mathcal{D}_{\mathcal{F}}$ for the $C^{0}$-topology such that $\mathcal{W}_{0} \subset \mathcal{W}, f^{ \pm 1} \circ g \in \mathcal{W}$ and $P\left(f^{ \pm 1} \circ g\right)=$ $P(f)^{ \pm 1} P(g)$ for all $f, g \in \mathcal{W}_{0}$. Let $\mathcal{U}$ be a path-connected neighborhood of the identity for the topology $\mathcal{T}$ contained in $\mathcal{W}_{0}$. Consider an open neighborhood $\mathcal{U}_{0}$ of the identity for the topology $\mathcal{T}$ such that $f^{ \pm 1} \circ g \in \mathcal{U}$ for all $f, g \in \mathcal{U}_{0}$.

Clearly $\left\{g \mathcal{U}_{0}\right\}_{g \in \mathcal{D}_{\mathcal{F}}}$ is an open cover of $\mathcal{D}_{\mathcal{F}}$ for the topology $\mathcal{T}$. Since $\mathcal{T}$ is second countable, it admits a countable subcover $\left\{g_{n} \mathcal{U}_{0}\right\}_{n \geq 1}$. The set $P\left(\mathcal{W}_{0}\right)$ is uncountable by Lemma 2.10 and equal to the union $\cup_{n=1}^{\infty} P\left(g_{n} \mathcal{U}_{0} \cap \mathcal{W}_{0}\right)$. Hence there exists $n_{0} \in \mathbb{N}$ such that $P\left(g_{n_{0}} \mathcal{U}_{0} \cap \mathcal{W}_{0}\right)$ is uncountable. Notice that $f^{-1} \circ g$ belongs to $\mathcal{U}$ for all $f, g \in g_{n_{0}} \mathcal{U}_{0}$ and thus our choices of $\mathcal{U}$ and $\mathcal{W}_{0}$ imply that $P(\mathcal{U})$ is uncountable. Since $\mathcal{U}$ is path-connected for the topology $\mathcal{T}$, that is finer than the $C^{0}$-topology, we deduce that $P_{\mid \mathcal{U}}$ is constant by Corollary 2.14 , obtaining a contradiction.

Remark 2.16 The group $\mathcal{D}_{\mathcal{F}}$ has no natural structure of Lie group. Indeed, local pathconnectedness is automatic if $\mathcal{D}_{\mathcal{F}}$ is a Lie group modeled in a local Fréchet space. Moreover, requiring the topology of $\mathcal{D}_{\mathcal{F}}$ to be finer than the $C^{0}$-topology and second countable is appropriate, since $\mathcal{D}_{\mathcal{F}}$ is a group of transformations of a compact manifold.

Remark 2.17 As a corollary of Theorem 2.9, we deduce that the group $\mathcal{D}_{\mathcal{F}}$ is not locally path-connected for the $C^{r}$-topology for any $0 \leq r \leq \infty$.

Remark 2.18 Theorem 2.9 provides an example of a closed subgroup of $\mathcal{D}\left(T^{2}\right)$ for which the LPSAC-topology and the induced topology do not coincide. More precisely, the induced topology of the subgroup is not LPSAC and its LPSAC-topology is not countable. This shows in particular that condition b) in Proposition 2.6 is not always fulfilled. 


\section{Deformations of smooth foliations and Kuranishi properties}

\subsection{The Kuranishi property for foliations}

In this section, we discuss deformations of smooth foliations. We emphasize that there are no general results concerning the existence of local moduli spaces for a smooth foliation. We show that having an automorphism group which is not locally path-connected is an obstruction to the existence of a smooth local moduli space in the sense of Definition 3.1. Hence, the example of Sect. 2 does not admit one.

Throughout this section, we use the notations introduced at the beginning of Sect. 2. We first review the notion of deformation of a smooth foliation and its encoding by vectorial forms. For omitted details, we refer to [11].

Let $\mathcal{E}$ be the Fréchet manifold of $p$-dimensional distributions of $T M$, that is smooth sections of the Grassmannian of $p$-dimensional subspaces of $T M$. Let $\mathcal{I}$ be the closed subset of involutive distributions so defining foliations. Fix a foliation $\mathcal{F}$, i.e. fix $T \mathcal{F}$ in $\mathcal{I}$.

A Fréchet local chart of $\mathcal{E}$ in a neighborhood of $T \mathcal{F}$ is given by a neighborhood of 0 in the Fréchet space of smooth 1 -forms on $T \mathcal{F}$ with values in $N \mathcal{F}$. Such a form $\omega$ corresponds to the distribution whose value at $x \in M$ is the subspace $\left\{v+\omega(v) \mid v \in T_{x} \mathcal{F}\right\}$. Such a form encodes an involutive distribution if and only if it satisfies an explicit quadratic equation, see $[11, \S 2]$.

The Fréchet Lie group $\mathcal{D}$ acts smoothly on the right on $\mathcal{E}$ by pull-backs. This action preserves $\mathcal{I}$. The isotropy group of $T \mathcal{F}$ is $\mathcal{D}_{\mathcal{F}}$.

The tangent space of $\mathcal{D}$ at identity $e$ is the Lie algebra of smooth vector fields $\mathfrak{X}$. We fix a Fréchet chart $\zeta$ from a neighborhood of 0 in $\mathfrak{X}$ onto a neighborhood of $e$ in $\mathcal{D}$, cf. Remark 2.2 a).

We look for a smooth local section $T$ to the action of $\mathcal{D}$ at $T \mathcal{F}$. Such a local section encodes all distributions close enough to $T \mathcal{F}$ up to isomorphisms. But we also ask for good properties of minimality of the section, that is we also want the intersection of the section with the local orbit at $T \mathcal{F}$ to be as small as possible. More precisely, we set

Definition 3.1 We say the the foliation $\mathcal{F}$ has the Kuranishi property if there is a smooth Fréchet submanifold $T$ of $\mathcal{E}$ passing through $T \mathcal{F}$ which fulfills the following properties

(a) There exist a neighborhood $U$ of $T \mathcal{F}$ in $\mathcal{E}$, a vector subspace $\mathfrak{L}$ of $\mathfrak{X}$ and a neighborhood $V$ of 0 in $\mathfrak{L}$ such that the mapping

$$
\Phi:(\xi, \omega) \in V \times T \longmapsto \omega \cdot \zeta(\xi) \in U
$$

is a Fréchet isomorphism.

(b) There exists a neighborhood $W$ of $e$ in $\mathcal{D}$ such that the intersection of $T$ with the local orbit $T \mathcal{F} \cdot W$ is reduced to the point $T \mathcal{F} \in T$.

Remarks 3.2 (a) The first condition implies that $T$ parametrizes all the distributions close to $T \mathcal{F}$ up to isomorphism; the second one is a minimality condition.

(b) The restriction of $\Phi$ to $V \times(T \cap \mathcal{I})$ maps bijectively onto $U \cap \mathcal{I}$. Hence $T \cap \mathcal{I}$ parametrizes all the foliations close to $\mathcal{F}$ up to isomorphism.

(c) The second condition is exactly condition (MC2) in [19].

(d) Definition 3.1 is modeled on Kuranishi's theorem for deformations of compact complex manifolds as it is proved in [21, §4.3]. The above Kuranishi property is pertinent for any type of geometric structures that can be encoded by elements of a Fréchet space acted on by a subgroup of $\mathcal{D}$ such that isomorphic structures corresponds to elements in 
the same orbit. In fact, besides complex structures, the following geometric structures always have the Kuranishi property: transversely holomorphic foliations [7], Riemannian metrics [5], anti-self-dual (ASD) hermitian connections on 4-manifolds [3]. More detailed considerations about the notion of Kuranishi type properties can be found in [19].

The next proposition relates $\mathcal{D}, \mathcal{D}_{\mathcal{F}}$ and $V$.

Proposition 3.3 Assume that a foliation $\mathcal{F}$ has the Kuranishi property. Then the germ of $\mathcal{D}$ at $\mathrm{Id}$ is homeomorphic to the germ of $\mathcal{D}_{\mathcal{F}} \times \zeta(V)$ at $(\mathrm{Id}, \mathrm{Id})$. Moreover, if $\mathcal{D}_{\mathcal{F}}$ is a Fréchet submanifold of $\mathcal{D}$ then the germs $\left(\mathcal{D}\right.$, Id) and $\left(\mathcal{D}_{\mathcal{F}} \times \zeta(V)\right.$, (Id, Id)) are Fréchet isomorphic.

Proof Consider the map $F: \mathcal{D}_{\mathcal{F}} \times \zeta(V) \rightarrow \mathcal{D}$ defined by $F(f, g)=f \circ g$. Let us define a local inverse. Consider the smooth map $G_{1}:(\mathcal{D}, \mathrm{Id}) \rightarrow(\mathcal{E}, T \mathcal{F})$ defined by $G_{1}(h)=T \mathcal{F} \cdot h$. Let $G_{2}: \mathcal{E} \rightarrow \mathcal{D}$ be the smooth map defined by $G_{2}=\zeta \circ \pi_{1} \circ \Phi^{-1}$. It satisfies that the map $b(h)=\left(G_{2} \circ G_{1}\right)(h)$ is the unique element of $\zeta(V)$ such that $T \mathcal{F} \cdot h=T \mathcal{F} \cdot b(h)$ for any $h \in \mathcal{D}$ in a neighborhood of Id. We define $a(h)=h \circ b(h)^{-1}$, it belongs to $\mathcal{D}_{\mathcal{F}}$ by construction. As a consequence, the map $G(h)=(a(h), b(h))$ is a smooth map from $\mathcal{D}$ to $\mathcal{D}_{\mathcal{F}} \times \zeta(V)$. It is clear that $G$ is the local inverse of $F$.

Since $\mathcal{D}$ is locally path-connected, we deduce

Corollary 3.4 Assume that the foliation $\mathcal{F}$ has the Kuranishi property. Then the isotropy group $\mathcal{D}_{\mathcal{F}}$ is locally path-connected.

From this result and Remark 2.17, we obtain

Corollary 3.5 The foliation constructed in Sect. 2 does not have the Kuranishi property.

\subsection{The Kuranishi property for diffeomorphisms of the circle}

Let us study the Kuranishi property for foliations of the 2-torus $T^{2}$ defined by suspension of diffeomorphisms of the circle as described in Sect. 2. The foliations in a neighborhood of a suspension are classified by the moduli space of their holonomy maps: the foliations are $C^{\infty}$-conjugated if and only if the holonomy maps are $C^{\infty}$-conjugated. As a consequence, it is natural to study the Kuranishi property for diffeomorphisms of the circle.

Remark 3.6 Proposition 3.3 and Corollary 3.4 can be immediately generalized for $\mathcal{D}^{\infty}\left(S^{1}\right)$ where the action by pull-back is replaced by the action by conjugacy. The isotropy group $\mathcal{D}_{\mathcal{F}}$ has to be replaced with the centralizer $Z^{\infty}(h)$ in the version of Proposition 3.3 for diffeomorphisms. The analogue of Corollary 3.4 for diffeomorphisms implies that if $h \in$ $\mathcal{D}^{\infty}\left(S^{1}\right)$ has the Kuranishi property then $Z^{\infty}(h)$ is locally path-connected. In particular the diffeomorphism $h_{0} \in \mathcal{D}^{\infty}\left(S^{1}\right)$ given by Corollary 2.14 does not satisfy the Kuranishi property.

If $h \in \mathcal{D}^{\infty}\left(S^{1}\right)$ satisfies the Kuranishi property then the smallest local orbit in the neighborhood of $h$ is the local orbit of $h$. We interpret this principle in terms of the centralizer of $h$.

Proposition 3.7 Assume that the diffeomorphism $h$ has the Kuranishi property and $Z^{\infty}(h)$ is discrete. Then $Z^{\infty}(g)$ is discrete for any $g$ in a neighborhood of $h$ in $\mathcal{D}^{\infty}\left(S^{1}\right)$.

Proof The proof of Proposition 3.3 implies that $\zeta(V)$ contains a neighborhood of Id in $\mathcal{D}^{\infty}\left(S^{1}\right)$. Since $\Phi$ is injective, every element $g$ of $T$ in a neighborhood of $h$ satisfies that 
$Z^{\infty}(g)$ is discrete. Moreover, $T$ intersects all local orbits of elements of $\mathcal{D}^{\infty}\left(S^{1}\right)$ defined in a neighborhood of $h$ and hence $Z^{\infty}(g)$ is discrete for any $g$ in a neighborhood of $h$ in $\mathcal{D}^{\infty}\left(S^{1}\right)$.

Proposition 3.8 Assume that $h \in \mathcal{D}^{\infty}\left(S^{1}\right)$ has the Kuranishi property. Moreover, suppose that $\left(Z^{\infty}(h), \mathrm{Id}\right)$ is the germ of a finite dimensional manifold of dimension $k$. Then there exists a neighborhood $U$ of $h$ in $\mathcal{D}^{\infty}\left(S^{1}\right)$ such that the centralizer $Z^{\infty}(g)$ does not contain a manifold of dimension greater than $k$ for any $g \in U$.

Proof We consider the notations in Definition 3.1. The manifold $\zeta(V)$ has codimension $k$ in $\mathcal{D}^{\infty}\left(S^{1}\right)$ by Proposition 3.3. Suppose that there exist $g \in U$ and a germ of manifold $(N$, Id) of dimension greater than $k$ and contained in $Z^{\infty}(g)$. We can suppose $g \in T$ without loss of generality. The tangent spaces of $N$ and $\zeta(V)$ at Id contain a common vector $X \neq 0$. Since $f^{-1} \circ g \circ f=g$ for any $f \in N$, the vector $X$ is in the kernel of the differential of the map $f \mapsto f^{-1} \circ g \circ f$ at Id. But such differential is injective since $\Phi$ is an isomorphism. We obtain a contradiction.

Corollary 3.4 and Propositions 3.7 and 3.8 provide negative criteria for the Kuranishi property. Let us introduce a new one.

Proposition 3.9 Assume that $h \in \mathcal{D}^{\infty}\left(S^{1}\right)$ has the Kuranishi property. Moreover, suppose that $\left(Z^{\infty}(h), \mathrm{Id}\right)$ is the germ of a one dimensional manifold. Then there exists a neighborhood $U$ of $h$ in $\mathcal{D}^{\infty}\left(S^{1}\right)$ such that the set $\mathfrak{D}:=\left\{g \in U: Z^{\infty}(g)\right.$ isdiscrete $\}$ is open.

Proof We consider the notations in Definition 3.1. Notice that if $g, g^{\prime} \in U$ belong to the same orbit then $g$ belongs to $\mathfrak{D}$ if and only if $g^{\prime}$ does. So given $g \in \mathfrak{D}$ we can replace it with some other diffeomorphism in the orbit of $g$ without lack of generality, for instance we can suppose $g \in T$.

Let $g \in \mathfrak{D}$. Since $Z^{\infty}(g)$ is discrete, the map $A: \mathcal{D}^{\infty}\left(S^{1}\right) \rightarrow \mathcal{D}^{\infty}\left(S^{1}\right)$ defined by $A(f)=f^{-1} \circ g \circ f$ is injective in a neighborhood of Id. Let us consider $f$ in a neighborhood of Id in $Z^{\infty}(h)$ such that $A(f)$ belongs to the "plaque" $A(\zeta(V))$. Then $A(f)=A\left(f^{\prime}\right)$ for some $f^{\prime} \in \zeta(V)$ in a neighborhood of Id. We obtain $f^{\prime}=f$ if $f$ is in a small neighborhood of Id by injectivity of $A$. Since $f \in Z^{\infty}(h), f^{\prime} \in \zeta(V)$ and the expression of an element of $\mathcal{D}^{\infty}\left(S^{1}\right)$ as a product of an element of $Z^{\infty}(h)$ and an element of $\zeta(V)$ is unique by Proposition 3.3, we obtain $f=\operatorname{Id}$ and $f^{\prime}=\mathrm{Id}$. We deduce that $A(f)$ does not belong to the "plaque" $A(\zeta(V))$ for any $f \in Z^{\infty}(h) \backslash\{I d\}$ in a neighborhood of Id. In particular the map $B:=\pi_{2} \circ \Phi^{-1} \circ A_{\mid Z^{\infty}(h)}$ is non-constant. Notice that $B(f)$ belongs to $T$ and satisfies $B(f)=b(f)^{-1} \circ g \circ b(f)$ where $b(f):=f \circ\left[\left(\zeta \circ \pi_{1} \circ \Phi^{-1} \circ A\right)(f)\right]^{-1}$ and the map $b: Z^{\infty}(h) \rightarrow \mathcal{D}^{\infty}\left(S^{1}\right)$ is smooth.

Since $B$ is non-constant in every neighborhood of Id in the one-dimensional manifold $Z^{\infty}(h)$, we can consider a point $f_{0}$ in a neighborhood of Id such that the differentials of $B$ (and $b$ ) at $f_{0}$ have rank 1 . The equation

$\left(b\left(f_{0}\right)^{-1} \circ b(f)\right)^{-1} \circ\left(b\left(f_{0}\right)^{-1} \circ g \circ b\left(f_{0}\right)\right) \circ\left(b\left(f_{0}\right)^{-1} \circ b(f)\right)=b(f)^{-1} \circ g \circ b(f)=B(f)$

implies

$$
\left(b\left(f_{0}\right)^{-1} \circ b\left(f_{0} \circ f\right)\right)^{-1} \circ\left(b\left(f_{0}\right)^{-1} \circ g \circ b\left(f_{0}\right)\right) \circ\left(b\left(f_{0}\right)^{-1} \circ b\left(f_{0} \circ f\right)\right)=B\left(f_{0} \circ f\right) .
$$

We can suppose that the differentials of $B(f)$ and $b(f)$ have rank 1 at Id up to replace $b(f)$, $B(f)$ and $g$ by $b\left(f_{0}\right)^{-1} \circ b\left(f_{0} \circ f\right), B\left(f_{0} \circ f\right)$ and $A\left(b\left(f_{0}\right)\right)$ respectively. By parametrizing 
$\left(Z^{\infty}(h), \mathrm{Id}\right)$ with $(\mathbb{R}, 0)$ let us denote $b(t)$ and $B(t)$ instead of $b(f)$ and $B(f)$ where $t$ is a real parameter.

Consider the smooth map $\hat{C}: \mathbb{R} \times T \rightarrow T$ defined in a neighborhood of $(0, g)$ in $\mathbb{R} \times T$ by the formula $\hat{C}\left(t, g^{\prime}\right)=\left(\pi_{2} \circ \Phi^{-1}\right)\left(b(t)^{-1} \circ g^{\prime} \circ b(t)\right)$. It satisfies $\hat{C}(t, g) \equiv B(t)$ and hence the differential of $\hat{C}(\cdot, g)$ has rank 1 at 0 . Let $g^{\prime}$ be an element of $T$ in a small neighborhood of $g$. The map $C: \mathbb{R} \rightarrow T$ defined by $C(t):=\hat{C}\left(t, g^{\prime}\right)$ is an immersion at 0 . In particular $C$ is injective in a neighborhood of 0 . The formula

$$
c(t):=b(t) \circ\left[\left(\zeta \circ \pi_{1} \circ \Phi^{-1}\left(b(t)^{-1} \circ g^{\prime} \cdot b(t)\right)\right]^{-1}\right.
$$

defines a smooth map such that $c(t)^{-1} \circ g^{\prime} \circ c(t) \equiv C(t)$. Since $C(t)$ is an immersion and $\Phi$ is an isomorphism, $c(t)$ is transversal to $\zeta(V)$ at $t=0$. Consider the smooth maps $d: \mathbb{R} \times \zeta(V) \rightarrow \mathcal{D}^{\infty}\left(S^{1}\right)$ and $D: \mathbb{R} \times \zeta(V) \rightarrow \mathcal{D}^{\infty}\left(S^{1}\right)$ defined by $d(t, f)=c(t) \circ f$ and $D(t, f)=d(t, f)^{-1} \circ g^{\prime} \circ d(t, f)$ respectively. Since $\Phi$ is injective, the map $D$ is injective in a neighborhood of $(0, \mathrm{Id})$ and so is $d$. Assume for now that $\operatorname{Im}(d)$ contains a neighborhood of Id in $\mathcal{D}^{\infty}\left(S^{1}\right)$. Therefore the map $f \mapsto f^{-1} \circ g^{\prime} \circ f$ is injective in a neighborhood of Id in $\mathcal{D}^{\infty}\left(S^{1}\right)$ and hence $Z^{\infty}\left(g^{\prime}\right)$ is discrete.

Finally let us show that $\operatorname{Im}(d)$ contains a neighborhood of $\operatorname{Id}$ in $\mathcal{D}^{\infty}\left(S^{1}\right)$. There exists a local smooth submersion $\alpha: \mathcal{D}^{\infty}\left(S^{1}\right) \rightarrow \mathbb{R}$ defined by the formula $\alpha(f)=\left(\pi_{1} \circ G\right)(f)$ where $G$ is the Fréchet isomorphism defined in the proof of Proposition 3.3. By definition, we have $\alpha^{-1}(0)=\zeta(V)$. Let us define the smooth function $\beta: \mathbb{R} \times \mathcal{D}^{\infty}\left(S^{1}\right) \rightarrow \mathbb{R}$ by $\beta(t, f)=\alpha\left(c(t)^{-1} \circ f\right)$. The image by $t \mapsto \beta(t, \mathrm{Id})$ of any neighborhood of 0 in $\mathbb{R}$ contains a neighborhood of 0 by the transversality of $c(t)$ and $\zeta(V)$ at $t=0$. In particular there exists $t_{0} \in \mathbb{R}^{+}$near 0 such that $\beta\left(t_{0}, f\right) \beta\left(-t_{0}, f\right)<0$ for any $f$ in a neighborhood of Id in $\mathcal{D}$. Fix a small neighborhood $U^{\prime}$ of Id in $\mathcal{D}$. Given $f \in U^{\prime}$, Bolzano's theorem assures the existence of $t_{f} \in\left(-t_{0}, t_{0}\right)$ such that $\beta\left(t_{f}, f\right)=0$. This implies $c\left(t_{f}\right)^{-1} \circ f \in \zeta(V)$ and hence $f=d\left(t_{f}, c\left(t_{f}\right)^{-1} \circ f\right) \in \operatorname{Im}(d)$.

Let us generalize Corollary 3.5 to any diffeomorphism of the circle with irrational rotation number. We denote by $\mathcal{D}_{\alpha}^{\infty}\left(S^{1}\right)$ the set of $C^{\infty}$-diffeomorphisms of rotation number $\alpha$.

Proposition 3.10 Assume that $h \in \mathcal{D}^{\infty}\left(S^{1}\right)$ has irrational rotation number. Then $h$ does not satisfy the Kuranishi property.

Proof Consider the notations in Definition 3.1. Suppose, aiming at contradiction, that $h$ satisfies the Kuranishi property. First, we consider the case where $h$ is not $C^{\infty}$-conjugated to a rotation. Analogously as in the proof of Corollary 2.14, the path-connected component of Id in $Z^{\infty}(h)$ is equal to $\{\operatorname{Id}\}$. Hence $Z^{\infty}(h)$ is discrete by the analogue of Corollary 3.4 for diffeomorphisms. The set of diffeomorphisms $C^{\infty}$-conjugated to $R_{\rho(h)}$ is dense in $\mathcal{D}_{\rho(h)}^{\infty}\left(S^{1}\right)$ by a theorem of Yoccoz [29, Chapitre III]. This contradicts Proposition 3.7 since $Z^{\infty}(g)$ is $C^{\infty}$-conjugated to $Z^{\infty}\left(R_{\rho(h)}\right)=\left\{R_{\alpha}: \alpha \in S^{1}\right\}$ and hence non-discrete if $g$ is $C^{\infty}$ conjugated to the rotation $R_{\rho(h)}$.

Suppose that $h$ is $C^{\infty}$-conjugated to the rotation $R_{\alpha}$ where $\alpha=\rho(h)$. Indeed we can suppose $h=R_{\alpha}$ without lack of generality. We claim that the diffeomorphism $R_{p / q}$ has an infinite dimensional centralizer for any rational rotation number $p / q$ where $p$ and $q$ are coprime integer numbers. Indeed, given the $q$-to-one covering map $\sigma: S^{1} \rightarrow S^{1}$ defined by $\sigma(z)=z^{q}$ (we consider that $S^{1}$ is embedded in $\mathbb{C}$ ), we have that $Z^{\infty}\left(R_{p / q}\right)$ is the group of lifts of diffeomorphisms of the circle with respect to the covering map $\sigma$ and hence infinite dimensional. Since there are rational rotations in every neighborhood of $R_{\alpha}$ and $\operatorname{dim} Z^{\infty}\left(R_{\alpha}\right)=1$, this contradicts Proposition 3.8. 
Remark 3.11 There are diffeomorphisms of $S^{1}$ with rational rotation number that do not satisfy the Kuranishi property. Consider the subset $D_{p / q}$ of $\mathcal{D}^{\infty}\left(S^{1}\right)$ of diffeomorphisms with rational rotation number $p / q$ and a unique finite orbit. Let $E_{p / q}$ be the subset of $D_{p / q}$ of diffeomorphisms $h$ such that $Z^{\infty}(h)=\langle h\rangle$; it is a dense open subset of $D_{p / q}$ by a theorem of Kopell [14]. Fix $h \in E_{p / q}$, clearly $Z^{\infty}(h)$ is discrete. We show below that there are elements of $\mathcal{D}^{\infty}\left(S^{1}\right)$ in any neighborhood of $h$ whose centralizer is non-discrete. This implies that $h$ does not satisfy the Kuranishi property by Proposition 3.7. Moreover, since there are elements of $\cup_{\delta \in \mathbb{Q}} D_{\delta}$ (and then of $\cup_{\delta \in \mathbb{Q}} E_{\delta}$ ) in any neighborhood of an irrational rotation $R_{\alpha}$ and $\operatorname{dim} Z^{\infty}\left(R_{\alpha}\right)=1$, Proposition 3.9 shows in an alternative way that $R_{\alpha}$ does not satisfy the Kuranishi property.

Let us define $h_{\lambda}=R_{\lambda} \circ h$ for $\lambda \in \mathbb{R}$. Since $h$ has a unique finite orbit, and up to replace $h$ by $h^{-1}$ if necessary, we can suppose that $h$ has a lift $\tilde{h}$ to $\mathbb{R}$ such that $\operatorname{Fix}\left(\tilde{h}^{q}-p\right) \neq \varnothing$ and $\tilde{h}(x)-p / q \geq x$ for any $x \in \mathbb{R}$. As a consequence $h_{\lambda}^{q}$ does not have fixed points for $\lambda>0$ sufficiently small and hence $\rho\left(h_{\lambda}\right) \neq \rho(h)$. Since the rotation number varies continuously, given any $\epsilon>0$ there exists $\lambda \in(0, \epsilon)$ such that $h_{\lambda}$ has a diophantine rotation number and then is conjugated to a rotation and has a non-discrete centralizer.

The identity map satisfies the Kuranishi property. There are also non-trivial diffeomorphisms with rational rotation number that satisfy the Kuranishi property: the existence of a nice moduli space for the diffeomorphisms with rational rotation number whose periodic orbits are hyperbolic [29, XI.5.3] allows to find such examples.

Remark 3.12 Let us remark that the obstructions to the Kuranashi property provided by Corollary 3.4 and Propositions 3.7, 3.8 and 3.9 are related to the nature of centralizers and its dependence with respect to diffeomorphisms.

Observe that, if $h$ has the Kuranishi property, then its local orbit in $\mathcal{D}^{\infty}\left(S^{1}\right)$ must be closed. Having non-closed local orbit is thus the primary obstruction to having the Kuranishi property. However, it may be very difficult to check. Anyway, this idea can be used to provide an alternative proof of the lack of the Kuranishi property for every Liouville number $\alpha$ and any map $C^{\infty}$-conjugated to a rotation $R_{\alpha}$. Indeed Yoccoz's techniques [29, Chapitre III] allow to show that the closure of the local orbit $\left\{g^{-1} \circ R_{\alpha} \circ g: g \in W\right\}$ of $R_{\alpha}$ contains a neighborhood of $R_{\alpha}$ in $\mathcal{D}_{\alpha}^{\infty}\left(S^{1}\right)$ for any neighborhood $W$ of $\operatorname{Id}$ in $\mathcal{D}^{\infty}\left(S^{1}\right)$. Since the orbit of $R_{\alpha}$ is meager in $\mathcal{D}_{\alpha}^{\infty}\left(S^{1}\right)$ [12, Chapter XI, Théorème 4.3], the local orbit of $R_{\alpha}$ is non-closed.

It is worth mentioning, even if it is not a result about local orbits, that there exist a residual set $\mathcal{C}$ of Liouville numbers such that for any $\alpha \in \mathcal{C}$ every orbit in $\mathcal{D}_{\alpha}^{\infty}\left(S^{1}\right)$ is dense in $\mathcal{D}_{\alpha}^{\infty}\left(S^{1}\right)$ by a theorem of Benhenda [1] (whose generalization for any Liouville number has been recently announced by Avila and Krikorian). As a consequence we could choose the diffeomorphism $h_{0}$ provided by Corollary 2.14 to have a dense orbit in $\mathcal{D}_{\rho\left(h_{0}\right)}^{\infty}\left(S^{1}\right)$.

\subsection{Deformations of foliations on the 2-torus}

Let us study the relation between the Kuranishi property for a diffeomorphism of $S^{1}$ and the foliation of the 2-torus obtained by suspension of such a diffeomorphism. More precisely, given $h \in \mathcal{D}^{\infty}\left(S^{1}\right)$ we consider the torus $\mathbb{T}^{2} \sim \mathbb{R} \times S^{1} /\langle\tilde{h}\rangle$ where $\tilde{h}(t, x)=\left(t+1, h^{-1}(x)\right)$. The foliation $d x=0$ of $\mathbb{R} \times S^{1}$ defines a foliation $\mathcal{F}$ in the quotient $\mathbb{T}^{2}$ that is transverse to the fibration $d t=0$. The holonomy map of $\mathcal{F}$ along the path [0,1] (in the $t$-coordinate) in the transversal $t=0$ is equal to $h$. The main goal of the remainder of this section is proving the next proposition. 
Proposition 3.13 Suppose that hatisfies the Kuranishi property in $\mathcal{D}^{\infty}\left(S^{1}\right)$. Then $\mathcal{F}$ satisfies the Kuranishi property in the space $\mathcal{E}$ of $C^{\infty}$-foliations of dimension 1 of the torus.

Since $h$ satisfies the Kuranishi property, let $\Phi: V \times T \rightarrow U$ be the Fréchet isomorphism associated to $h$, where $V$ is a neighborhood of 0 in a subspace $\mathfrak{L}$ of $\mathfrak{X}\left(S^{1}\right), T$ is a Fréchet submanifold of $\mathcal{D}^{\infty}\left(S^{1}\right), U$ is a neighborhood of $h$ in $\mathcal{D}^{\infty}\left(S^{1}\right)$ and

$$
\Phi(\xi, g)=\zeta(\xi)^{-1} \circ g \circ \zeta(\xi) .
$$

First, let us explain how to construct a foliation with a prescribed holonomy map.

Lemma 3.14 There exists a $C^{\infty}$ map $s: T \rightarrow \mathcal{E}$, defined in a neighborhood of $h$ in $T$, such that $s(h)=\mathcal{F}$ and $s(g)$ is a $C^{\infty}$ foliation of $\mathbb{T}^{2}$ that is transverse to the fibration $d t=0$ and such that the holonomy of $s(g)$ associated to the path $t \in[0,1]$ and the transversal $t=0$ is equal to $g$ for any $g \in \mathcal{D}^{\infty}\left(S^{1}\right)$ in a neighborhood of $h$.

Proof The foliation $\mathcal{F}$ was defined in such a way that its lift to $\mathbb{R} \times S^{1}$ is equal to the foliation $d x=0$. In order to obtain new holonomy maps we are going to modify the foliation $d x=0$ in $\left\{(t, x) \in\left[t_{0}, t_{1}\right] \times S^{1}\right\}$ where we fix $0<t_{0}<t_{1}<1$. Let us consider a $C^{\infty}$ function $r: \mathbb{R} \rightarrow[0,1]$ such that $r(t)=0$ if $t \leq t_{0}$ and $r(t)=1$ if $t \geq t_{1}$. We denote by $\pi: \mathbb{R} \rightarrow S^{1}$ the covering map $\pi(y)=e^{2 \pi i y}$ where we identify $S^{1}$ with the unit circle of $\mathbb{C}$.

We fix a local chart $\tau: T \rightarrow E$ of $T$, where $E$ is a Fréchet space, such that $\tau(\mathrm{Id})=0$. The map $\mathbb{R} \times T \rightarrow T$ given by $(t, f) \mapsto f_{t}=\tau^{-1}(r(t) \cdot \tau(f))$ is well-defined and smooth. Let $\mathcal{X}^{t}\left(S^{1}\right)$ be the Fréchet space of time-dependent vector fields on $S^{1}$. The map $T \rightarrow \mathcal{X}^{t}\left(S^{1}\right)$, that associates to $f \in T$ the vector field $X_{t}^{f}=\frac{d}{d t} f_{t}$, is smooth. We define $\bar{s}: T \rightarrow \mathcal{X}\left(\mathbb{R} \times S^{1}\right)$ as the map sending a diffeomorphism $g$ of $S^{1}$ close to $h$ to the distribution $\bar{s}(g)$ in $\mathbb{R} \times S^{1}$ determined by the vector field $X^{g}:=\frac{\partial}{\partial t}+X_{t}^{f}$ where $f=h^{-1} \circ g$. Since the foliation $\mathcal{G}$ defined by $X^{g}$ coincides with $\mathcal{F}$ in the neighborhood of $t=0$ and $t=1$, it follows that $\mathcal{G}$ coincides with $\mathcal{F}$, and therefore $\mathcal{G}$ induces a foliation $s(g)$ of $\mathbb{T}^{2} \sim \mathbb{R} \times S^{1} /\langle\tilde{h}\rangle$. The foliation $s(g)$ is transverse to $t=0$, its holonomy map at $t=0$ is equal to $g=h \circ f$ and $s: T \rightarrow \mathcal{E}$ is a $C^{\infty}$ map. By construction we have $s(h)=\mathcal{F}$.

Proof of Proposition 3.13 We keep the previous notations. We denote by $P$ the set of diffeomorphisms $F$ of $\mathbb{T}^{2}$ that satisfy $t \circ F=t$ and $F_{\mid t=0} \in \zeta(V)$. We define $\hat{\Phi}: P \times s(T) \rightarrow \mathcal{E}$ by the formula

$$
\hat{\Phi}(F, s(f))=s(f) \cdot F \text {. }
$$

Clearly, it is a $C^{\infty}$ map. Next, we will construct its local inverse $\Psi$ in a neighborhood of $\mathcal{F}$. Consider $\mathcal{G} \in \mathcal{E}$ close to $\mathcal{F}$. Let $g$ be the holonomy map associated to the foliation $\mathcal{G}$, the path $t \in[0,1]$ and the transversal $t=0$. Denote $\left(X, g_{0}\right)=\Phi^{-1}(g)$. By definition of $\Phi$, the holonomies $g$ and $g_{0}$ of $\mathcal{G}$ and $s\left(g_{0}\right)$ respectively, are conjugated by $\zeta(X)$. We claim that there exists a unique $F_{\mathcal{G}, s\left(g_{0}\right)}^{\zeta(X)} \in P$ such that $s\left(g_{0}\right) \cdot F_{\mathcal{G}, s\left(g_{0}\right)}^{\zeta(X)}=\mathcal{G}$ and $\left(F_{\mathcal{G}, s\left(g_{0}\right)}^{\zeta(X)}\right)_{\mid t=0}=\zeta(X)$. More precisely, let $H_{t_{0}}^{\mathcal{G}}$ be the holonomy map of the foliation $\mathcal{G}$, between the transversals $t=0$ and $t=t_{0}$ and associated to the path $t \in\left[0, t_{0}\right]$. We have

$$
F_{\mathcal{G}, s\left(g_{0}\right)}^{\zeta(X)}(t, x)=\left(t, H_{t}^{s\left(g_{0}\right)} \circ \zeta(X) \circ\left(H_{t}^{\mathcal{G}}\right)^{-1}(t, x)\right) .
$$

The map $\Psi: \mathcal{E} \rightarrow P \times s(T)$ defined by

$$
\Psi(\mathcal{G})=\left(F_{\mathcal{G}, s\left(g_{0}\right)}^{\zeta(X)},\left(s \circ \pi_{2} \circ \Phi^{-1}\right)(g)\right)
$$


is $C^{\infty}$ and it is a local inverse of $\hat{\Phi}$ in a neighborhood of $\mathcal{F}$. Hence $\hat{\Phi}$ is a local Fréchet isomorphism in a neighborhood of (Id, $\mathcal{F})$.

It remains to show that the local orbit of $\mathcal{F}$ coincides with $\mathcal{F} \cdot P$. Let $\mathcal{G}$ be a foliation in the local orbit of $\mathcal{F}$. Then the holonomy $g$ of $\mathcal{G}$, associated to the path $t \in[0,1]$ and the transversal $t=0$, is in the local orbit of $h$. Thus there exists a unique $f \in \zeta(V)$ such that $f^{-1} \circ h \circ f=g$ as a consequence of the Kuranishi property for $h$. Since $F_{\mathcal{G}, \mathcal{F}}^{f} \in P$ and $\mathcal{F} \cdot F_{\mathcal{G}, \mathcal{F}}^{f}=\mathcal{G}$ we are done.

Remark 3.15 We do not know if the converse of Proposition 3.13 is true. The map $\hat{\Phi}$ : $P \times s(T) \rightarrow \mathcal{E}$ used to prove the Kuranishi property has additional properties. It satisfies that if two foliations $\mathcal{G}$ and $\mathcal{G}^{\prime}$ have the same holonomy $g$ then both are in the same "plaque", i.e. there exists $\mathcal{F}^{\prime} \in s(T)$ and $F, F^{\prime} \in P$ such that $\mathcal{F}^{\prime} \cdot F=\mathcal{G}$ and $\mathcal{F}^{\prime} \cdot F^{\prime}=\mathcal{G}^{\prime}$. Moreover, the "transversal actions" $F_{\mid t=0}$ and $F_{\mid t=0}^{\prime}$, induced in the corresponding leaf spaces, coincide. As a consequence, the Kuranishi property for diffeomorphisms of the circle is equivalent to a stronger version of the Kuranishi property for foliations of the torus. Showing that the Kuranishi property for foliations is equivalent to this stronger version is the main difficulty to show that the Kuranishi property for foliations implies its analogous counterpart for diffeomorphisms.

\section{The automorphism group of a transversely holomorphic foliation}

Throughout this section we assume that the foliation $\mathcal{F}$ on the compact manifold $M$ is transversely holomorphic, of (real) dimension $p$ and complex codimension $q$. This means that the atlas of adapted local charts $\left\{\left(U_{i}, \varphi_{i}\right)\right\}$ can be chosen as taking values in $\mathbb{R}^{p} \times \mathbb{C}^{q}$ and that the maps $\left\{\gamma_{i j}\right\}$ fulfilling the cocycle condition (1) are local holomorphic transformations of $\mathbb{C}^{q}$.

The transverse complex structure of $\mathcal{F}$ induces a complex structure on the normal bundle $\nu \mathcal{F}=T M / T \mathcal{F}$ of the foliation, which is invariant by the holonomy of the foliation.

We denote by $\operatorname{Aut}(\mathcal{F})$ the group of automorphisms of the foliation. That is, $\operatorname{Aut}(\mathcal{F})$ is the group of elements $f \in \mathcal{D}_{\mathcal{F}}$ which are transversely holomorphic in the sense that, in adapted local coordinates they are of the form

$$
f(x, z)=\left(f_{1}(x, z, \bar{z}), f_{2}(z)\right)
$$

with $f_{2}$ holomorphic transformations.

Let $\mathfrak{a u t}(\mathcal{F})$ denote the Lie algebra of (real) vector fields $\xi$ on $M$ whose flows are oneparameter subgroups of $\operatorname{Aut}(\mathcal{F})$. In particular, $\mathfrak{a u t}(\mathcal{F})$ is a Lie subalgebra of $\mathfrak{X}_{\mathcal{F}}$.

In adapted local coordinates $(x, z)$, a (real) vector field $\xi \in \mathfrak{X}$ is written in the form

$$
\xi=\sum_{i} a^{i}(x, z, \bar{z}) \frac{\partial}{\partial x^{i}}+\sum_{j} b^{j}(x, z, \bar{z}) \frac{\partial}{\partial z^{j}}+\sum_{j} \bar{b}^{j}(x, z, \bar{z}) \frac{\partial}{\partial \bar{z}^{j}},
$$

where the functions $a^{i}$ are real whereas $b^{j}$ are complex-valued. Then $\xi$ is a foliated vector field, i.e. it belongs to $\mathfrak{X}_{\mathcal{F}}$, if $b^{j}$ are basic functions, that is if $b^{j}$ only depend on the coordinates $(z, \bar{z})$. And $\xi$ is a holomorphic foliated vector field, i.e. it belongs to $\mathfrak{a u t}(\mathcal{F})$, if $b^{j}$ are holomorphic basic functions.

Notice that $\mathcal{D}_{\mathcal{L}}$ is a normal subgroup of $\operatorname{Aut}(\mathcal{F})$ and that $\mathfrak{X}_{\mathcal{L}}$ is an ideal of $\mathfrak{a u t}(\mathcal{F})$. We denote by $\mathcal{G}=\mathfrak{a u t}(\mathcal{F}) / \mathfrak{X}_{\mathcal{L}}$ the quotient Lie algebra. Notice also that $\mathcal{D}_{\mathcal{L}}$ is not necessarily closed in $\operatorname{Aut}(\mathcal{F})$ as far as the leaves of $\mathcal{F}$ are not closed. 
We will suppose that a Riemannian metric $g$ on $M$, that will play an auxiliary role, has also been fixed.

We recall now some facts concerning transversely holomorphic foliations that we will use in the sequel (for the omitted details we refer to [4]). The complex structure on the normal bundle $\nu \mathcal{F}$, inherited from the transverse complex structure of $\mathcal{F}$, induces in the standard way a splitting of the complexified normal bundle

$$
v^{\mathbb{C}} \mathcal{F}=v^{1,0} \otimes v^{0,1} .
$$

There is a short exact sequence of complex vector bundles

$$
0 \longrightarrow E \longrightarrow T^{\mathbb{C}} M \stackrel{\pi^{1,0}}{\longrightarrow} v^{1,0} \longrightarrow 0
$$

where $\pi^{1,0}$ denotes the composition of natural projections

$$
T^{\mathbb{C}} M \rightarrow v^{\mathbb{C}} \mathcal{F} \rightarrow v^{1,0}
$$

and $E$ is the kernel of $\pi^{1,0}$. In local adapted coordinates $(x, z)$, the bundle $v^{1,0}$ is spanned by the (classes of) vector fields $\left[\partial / \partial z^{j}\right]$. In a similar way, the bundle $E$ is spanned by the vector fields $\partial / \partial x^{i}, \partial / \partial \bar{z}^{j}$, and the dual bundle $E^{*}$ is spanned by the (classes of) 1 -forms $\left[d x^{i}, d \bar{z}^{j}\right]$. For a given $k \in \mathbb{N}$, denote

$$
\mathcal{A}^{k}=\Gamma\left(\bigwedge^{k} E^{*} \otimes v^{1,0}\right) .
$$

The exterior derivative $d$ induces differential operators of order 1 (cf. [4])

$$
d_{\mathcal{F}}^{k}: \mathcal{A}^{k} \longrightarrow \mathcal{A}^{k+1} \text {. }
$$

In adapted local coordinates, the operators $d_{\mathcal{F}}^{k}$ act as follows

$$
\begin{aligned}
d_{\mathcal{F}}^{k}\left[\sum \alpha_{I J}^{\ell} d x^{I} \wedge d \bar{z}^{J} \otimes \frac{\partial}{\partial z^{\ell}}\right] & =\sum\left[d\left(\alpha_{I J}^{\ell} d x^{I} \wedge d \bar{z}^{J}\right)\right] \otimes\left[\frac{\partial}{\partial z^{\ell}}\right] \\
& =\sum\left[\left(\frac{\partial \alpha_{I J}^{\ell}}{\partial x^{i}} d x^{i}+\frac{\partial \alpha_{I J}^{\ell}}{\partial \bar{z}^{j}} d \bar{z}^{j}\right) \wedge d x^{I} \wedge d \bar{z}^{J} \otimes \frac{\partial}{\partial z^{\ell}}\right] .
\end{aligned}
$$

Notice that $\mathcal{A}^{0}$ is just the space of smooth sections of the vector bundle $v^{1,0}$ and that $\mathcal{G}$ is the kernel of the map $d_{\mathcal{F}}^{0}: \mathcal{A}^{0} \longrightarrow \mathcal{A}^{1}$. One has

Proposition 4.1 [4] The sequence

$$
0 \longrightarrow \mathcal{A}^{0} \stackrel{d_{\mathcal{F}}^{0}}{\longrightarrow} \mathcal{A}^{1} \stackrel{d_{\mathcal{F}}^{1}}{\longrightarrow} \mathcal{A}^{2} \stackrel{d_{\mathcal{F}}^{2}}{\longrightarrow} \cdots \longrightarrow \mathcal{A}^{p+q} \longrightarrow 0
$$

is an elliptic complex.

The elements of the quotient Lie algebra $\mathcal{G}=\mathfrak{a u t}(\mathcal{F}) / \mathfrak{X}_{\mathcal{L}}$ are naturally identified to the holomorphic basic sections of the normal bundle $v^{1,0}$, and are called holomorphic basic vector fields. In particular $\mathcal{G}$ is a complex Lie algebra in a natural way. Using the Riemannian metric on $M$, we can identify $\mathcal{G}$ to the vector space $\mathfrak{X}_{N}$ of those vector fields in $\mathfrak{X}$ that are orthogonal to $\mathcal{F}$. Notice that, although $\mathfrak{X}_{N}$ is not a Lie subalgebra of $\mathfrak{X}$, there is a vector space decomposition $\mathfrak{X}=\mathfrak{X}_{\mathcal{L}} \oplus \mathfrak{X}_{N}$.

As a corollary of Proposition 4.1 one obtains the following result, which was also proved by a different method by Gómez-Mont [8]. 
Proposition 4.2 (Gómez-Mont, and Duchamp and Kalka) Let $\mathcal{F}$ be a transversely holomorphic foliation on a compact manifold $M$. The Lie algebra $\mathcal{G}$ of holomorphic basic vector fields has finite dimension.

We denote by $G$ the simply-connected complex Lie group whose Lie algebra is $\mathcal{G}$.

Let $\mathcal{D}_{\mathcal{L}, 0}$ denote the connected component of $\mathcal{D}_{\mathcal{L}}$ containing the identity. Our main result concerning the structure of $\operatorname{Aut}(\mathcal{F})$ is the following

Theorem 4.3 Let $\mathcal{F}$ be a transversely holomorphic foliation on a compact manifold $M$. Endowed with the topology induced by $\mathcal{D}$, the group $\operatorname{Aut}(\mathcal{F})$ of automorphisms of $\mathcal{F}$ is a closed, strong ILH-Lie subgroup of $\mathcal{D}$ with Lie algebra $\mathfrak{a u t}(\mathcal{F})$. Moreover, the left cosets of the subgroup $\mathcal{D}_{\mathcal{L}, 0}$ define a Lie foliation $\mathcal{F}_{\mathcal{D}}$ on $\operatorname{Aut}(\mathcal{F})$, which is transversely modeled on the simply-connected complex Lie group $G$ associated to the Lie algebra $\mathcal{G}$.

We recall that a Lie foliation, modeled on a Lie group $G$, is defined by local submersions $\Phi_{i}$ with values in $G$ fulfilling $\Phi_{j}=L_{\gamma_{j i}} \circ \Phi_{i}$, where $\gamma_{i j}$ is a locally constant function with values in $G$ and $L_{\gamma_{i j}}$ denotes left translation by $\gamma_{i j}$.

The proof of the above theorem will be given in several steps:

(1) First, we use Theorem 2.5 to show that there is a strong ILH-Lie subgroup $\operatorname{Aut}^{\prime}(\mathcal{F})$ of $\mathcal{D}$, contained in $\operatorname{Aut}(\mathcal{F})$ and whose Lie algebra is $\mathfrak{a u t}(\mathcal{F})$. Note that this is not sufficient to assure that $\operatorname{Aut}^{\prime}(\mathcal{F})$ coincides with the connected component of the identity of $\operatorname{Aut}(\mathcal{F})$.

(2) We consider the group $\operatorname{Aut}(\mathcal{F})$ endowed with the topology induced by $\mathcal{D}$ and the group $\mathcal{D}_{\mathcal{L}}$ with the Fréchet topology given by Proposition 2.7. We find small neighborhoods of the identity $\mathcal{V}$ and $\mathcal{V}_{\mathcal{L}}$ of these two groups and we construct a continuous map $\Phi: \mathcal{V} \rightarrow \mathcal{D}$, with image $\tilde{\Sigma}=\Phi(\mathcal{V})$ contained in $\operatorname{Aut}(\mathcal{F})$, with the property that $\mathcal{V}$ is homeomorphic to the product $\mathcal{V}_{\mathcal{L}} \times \tilde{\Sigma}$.

(3) We show that $\tilde{\Sigma}$ is naturally identified to a neigbourhood $\Sigma$ of $e$ in the Lie group $G$. This implies that the induced topology of $\operatorname{Aut}(\mathcal{F})$ is LPSAC and reasoning as in the proof of Proposition 2.7 we conclude that, with the induced topology, $\operatorname{Aut}(\mathcal{F})$ is a closed strong ILH-Lie subgroup of $\mathcal{D}$, proving the first part of the Theorem.

(4) Finally, we prove that the map $\Phi$ is smooth with respect to the Fréchet structure of $\operatorname{Aut}(\mathcal{F})$ and that the identification of $\mathcal{V}$ with $\mathcal{V}_{\mathcal{L}} \times \Sigma$ is a local chart of $\operatorname{Aut}(\mathcal{F})$ defining the Lie foliation in a neighborhood of the identity. A similar construction of local charts around any element of $\operatorname{Aut}(\mathcal{F})$ completes the proof.

Thus we begin with showing the following statement.

Proposition 4.4 There is a strong ILH-Lie subgroup $\operatorname{Aut}^{\prime}(\mathcal{F})$ of $\mathcal{D}$ whose Lie algebra is $\mathfrak{a u t}(\mathcal{F})$. It is contained in $\operatorname{Aut}(\mathcal{F})$.

Proof Let $\pi: \mathfrak{X}=\Gamma(T M) \rightarrow \mathcal{A}^{0}=\Gamma\left(v^{1,0}\right)$ be the natural projection. That is, if $\xi \in \mathfrak{X}$ is written in local adapted coordinates as in (5), then

$$
\pi(\xi)=\sum_{j} b^{j}(x, z, \bar{z}) \frac{\partial}{\partial z^{j}} .
$$

Then $\pi$ is a differential operator of order 0 and its adjoint operator $\pi^{*}$, constructed using Riemannian metrics on $T M$ and $v^{1,0}$, is also a differential operator of order 0 such that $\pi \pi^{*}=$ Id.

We define the operator $A: \Gamma(T M) \rightarrow \mathcal{A}^{1}$ as the composition $A=d_{\mathcal{F}}^{0} \circ \pi$ and we set $B=d_{\mathcal{F}}^{1}$. Then one has $B A=0$ and $\operatorname{ker} A=\mathfrak{a u t}(\mathcal{F})$. Moreover the operator

$$
A A^{*}+B^{*} B=d_{\mathcal{F}}^{0} \circ \delta^{0}+\delta^{1} \circ d_{\mathcal{F}}^{1},
$$


where $\delta^{k}$ are formal adjoints of $d_{\mathcal{F}}^{k}$, is elliptic because of Proposition 4.1. Then Theorem 2.5 proves the existence of a strong ILH-Lie subgroup $\operatorname{Aut}^{\prime}(\mathcal{F})$ of $\mathcal{D}$ with Lie algebra is $\mathfrak{a u t}(\mathcal{F})$. The fact that $\operatorname{Aut}^{\prime}(\mathcal{F})$ is an integral submanifold of the distribution $T \mathcal{D}$ obtained by right translation of $\mathfrak{a u t}(\mathcal{F})$ implies that each smooth curve $c=c(t)$ in $\operatorname{Aut}^{\prime}(\mathcal{F})$ starting at the identity fulfills an equation of type

$$
\dot{c}(t)=\xi_{t} \circ c(t)
$$

where $\xi_{t}$ is a curve in $\mathfrak{a u t}(\mathcal{F})$. This implies that each diffeomorphism $c(t)$ preserves the transversely holomorphic foliation $\mathcal{F}$ showing that $\operatorname{Aut}^{\prime}(\mathcal{F})$ is contained in $\operatorname{Aut}(\mathcal{F})$.

Remark 4.5 The above argument also shows that the Sobolev completion of order $k, \operatorname{Aut}^{\prime k}(\mathcal{F})$, of $\operatorname{Aut}^{\prime}(\mathcal{F})$ is also a subgroup of the $\operatorname{Sobolev~completion~} \operatorname{Aut}^{k}(\mathcal{F})$ of $\operatorname{Aut}(\mathcal{F})$.

Proposition 4.6 Let us consider $\operatorname{Aut}(\mathcal{F})$ endowed with the topology induced by $\mathcal{D}$, and $\mathcal{D}_{\mathcal{L}}$ endowed with the Fréchet topology given by Proposition 2.7. There are open neighborhoods of the identity $\mathcal{V} \subset \operatorname{Aut}(\mathcal{F})$ and $\mathcal{V}_{\mathcal{L}} \subset \mathcal{D}_{\mathcal{L}}$, and a continuous map $\Phi: \mathcal{V} \rightarrow \mathcal{D}$, with image $\tilde{\Sigma}=\Phi(\mathcal{V})$ contained in $\operatorname{Aut}(\mathcal{F})$, such that the multiplication map

$$
\begin{aligned}
\Psi: \mathcal{V}_{\mathcal{L}} \times \tilde{\Sigma} & \longrightarrow \mathcal{V} \\
(f, s) & \mapsto f \circ s
\end{aligned}
$$

is a homeomorphism and the following diagram commutes

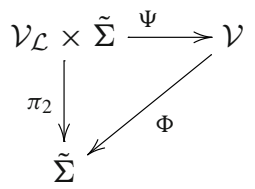

Proof The idea for proving this proposition is to associate, to any given diffeomorphism $f \in \operatorname{Aut}(\mathcal{F})$ close enough to the identity, the element $\bar{f} \in \operatorname{Aut}(\mathcal{F})$ fulfilling the following property: for each $w, \bar{f}(w)$ is the point of the local slice of the foliation through $f(w)$ which is at the minimal distance from $w$. The mapping $\bar{f}$ is uniquely determined by that condition and the diffeomorphisms $f$ and $\bar{f}$ differ by an element of $\mathcal{D}_{\mathcal{L}}$ close to the identity. Then $\tilde{\Sigma}$ will be the set of the diffeomorphisms $\bar{f}$ obtained in this way. In order to carry out this construction in a rigorous way we need to introduce appropriate families of adapted local charts.

We say that a local chart $(U, \varphi)$ of $M$ adapted to $\mathcal{F}$ is cubic if its image $\varphi(U) \subset \mathbb{R}^{p} \times \mathbb{C}^{q}$ is the product $C \times \Delta$ of a cube $C \subset \mathbb{R}^{p}$ and a polydisc $\Delta \subset \mathbb{C}^{q}$, and we say that it is centered at $w_{0} \in U$ if $\varphi\left(w_{0}\right)=(0,0)$. The submersion $\phi=\pi_{2} \circ \varphi$ maps $U$ onto $\Delta$ and the slices $S_{z}=\phi^{-1}(z)$ in $U$ are copies of $C$.

Let $N \mathcal{F} \cong \nu \mathcal{F}$ be the subbundle of $T M$ orthogonal to $T \mathcal{F}$ and let $p: N \mathcal{F} \rightarrow M$ be the natural projection. We denote by exp: $N \mathcal{F} \rightarrow M$ the geodesic exponential map associated to the Riemannian metric $g$ and we set $W_{\epsilon}=\{(w, v) \in N \mathcal{F}|| v \mid<\epsilon\}$. Since $M$ is compact there is $\epsilon_{1}>0$ such that $T_{w}=\exp \left(p^{-1}(w) \cap W_{\epsilon_{1}}\right)$ is a submanifold transverse to $\mathcal{F}$ for each $w \in M$.

Given a cubic adapted local chart $(U, \varphi)$, we set $W_{\epsilon, U}=W_{\epsilon} \cap p^{-1}(U)$ and we define $\psi_{U}: W_{\epsilon, U} \rightarrow \Delta \times M$ as the map

$$
\psi_{U}(w, v)=(\phi(w), \exp (w, v)) .
$$

Then there are constants $0<\epsilon_{0}<\epsilon_{1}$ and $0<\delta_{0}$ such that, if the diameter of $U$ fulfills diam $U<\delta_{0}$, then $\psi_{U}$ is a diffeomorphism from $W_{\epsilon_{0}, U}$ onto its image. We fix once and for 
all the values $\epsilon_{0}, \delta_{0}$ and we set $W_{U}=W_{\epsilon_{0}, U}$. We denote by $W_{U}^{z}$ the restriction of $W_{U}$ to the slice $S_{z}$, i.e. $W_{U}^{z}=\left\{(w, v) \in W_{U} \mid \phi(w)=z\right\}$.

Let $(U, \varphi)$ be a cubic adapted chart centered at $w_{0}$ and $U^{\prime}$ an open subset of $U$. We say that the pair $\left(U, U^{\prime}\right)$ is regular if the following conditions are fulfilled:

(R1) $\operatorname{diam} U<\delta_{0}$,

(R2) $w_{0} \in U^{\prime} \subset \bar{U}^{\prime} \subset U$ and $\left(U^{\prime},\left.\varphi\right|_{U^{\prime}}\right)$ is a cubic chart,

(R3) $\bar{U}^{\prime}$ is contained in the image $\exp \left(W_{U}^{z}\right)$ for each $z \in \Delta$.

If the pair $\left(U, U^{\prime}\right)$ is regular then the intersection of a slice $S_{z}$ of $U$ with $U^{\prime}$ is either empty or coincides with a slice of $U^{\prime}$. Condition (R3) implies that, for each point $w \in U^{\prime}$ and each slice $S_{z}$ of $U$ meeting $U^{\prime}$, there is a unique point $w^{\prime} \in S_{z}$ which is at the minimal distance between $w$ and $S_{z}$, namely

$$
w^{\prime}=p \circ \psi_{U}^{-1}(z, w) .
$$

Notice also that the submersion $\phi$ maps the intersection $T_{w}^{U^{\prime}}=T_{w} \cap U^{\prime}$ diffeomorphically onto the open polydisc $\phi\left(U^{\prime}\right) \subset \mathbb{C}^{q}$.

Since $M$ is compact, we can find positive numbers $0<\delta^{\prime}<\delta<\delta_{0}$, a finite family of adapted and cubic local charts $\left\{\left(U_{i}, \varphi_{i}\right)\right\}_{i=1, \ldots, m}$, centered at points $w_{i}$, and a family of 4-tuples

$$
\mathcal{U}=\left\{\left(U_{i}, U_{i}^{\prime}, V_{i}, V_{i}^{\prime}\right)\right\}_{i=1, \ldots, m}
$$

of open sets such that

(C1) $\left(U_{i}, U_{i}^{\prime}\right)$ is a regular pair fulfilling $\operatorname{diam} U_{i}<\delta$ and $\delta^{\prime}<d\left(w_{i}, \partial U_{i}^{\prime}\right)$,

(C2) $\bar{V}_{i} \subset U_{i}^{\prime},\left(V_{i},\left.\varphi_{i}\right|_{V_{i}}\right)$ is a cubic adapted local chart centered at $w_{i}$, diam $V_{i}<\delta^{\prime} / 4$ and $\left(V_{i}, V_{i}^{\prime}\right)$ is a regular pair,

(C3) the family $\left\{V_{i}^{\prime}\right\}_{i=1, \ldots, m}$ is an open covering of $M$.

We then set $\psi_{i}=\psi_{U_{i}}, W_{i}=W_{U_{i}}$ and $W_{i}^{z}=W_{U_{i}}^{z}$. We remark that such a covering has the following properties:

(P1) If $V_{i} \cap V_{j} \neq \emptyset$ then $\bar{V}_{i} \cup \bar{V}_{j} \subset U_{i}^{\prime} \cap U_{j}^{\prime}$,

(P2) given a point $w \in V_{i}^{\prime}$ and a slice $S_{z}$ in $U_{i}$ which meets $V_{i}$, the point $w^{\prime} \in S_{z}$ that is at the minimal distance from $w$ belongs to $V_{i}$.

We fix from now on such a family $\mathcal{U}$. We denote by $\mathcal{V}$ the open neighborhood of the identity in $\operatorname{Aut}(\mathcal{F})$ defined by $f \in \mathcal{V}$ if and only if $f\left(\bar{V}_{i}^{\prime}\right) \subset V_{i}$ for each $i=1, \ldots m$. We also define the subset $\mathcal{V}_{\mathcal{L}}$ of $\mathcal{D}_{\mathcal{L}}$ of those elements $f \in \mathcal{D}_{\mathcal{L}}$ fulfilling $f\left(\bar{V}_{i}^{\prime}\right) \subset V_{i}$ for each $i=1, \ldots m$ and keeping fixed each slice of $V_{i}^{\prime}$ (recall that a leaf of $\mathcal{F}$ can cut $V_{i}$ in many slices). The set $\mathcal{V}_{\mathcal{L}}$ is open in the Fréchet topology of $\mathcal{D}_{\mathcal{L}}$. We are now ready to define the map $\Phi: \mathcal{V} \rightarrow \operatorname{Aut}(\mathcal{F}) \subset \mathcal{D}$ and the set $\tilde{\Sigma}=\Phi(\mathcal{V})$.

Let $f$ be a fixed element in $\mathcal{V}$. For a given point $w \in M$ we choose $i=1, \ldots, m$ with $w \in V_{i}^{\prime}$. Then $\hat{w}=f(w) \in V_{i}$ and we denote by $S_{z}$ and $S_{\hat{z}}$ the slices of $U_{i}$ through the points $w$ and $\hat{w}$ respectively, i.e. $z=\phi_{i}(w)$ and $\hat{z}=\phi_{i}(f(w))$. Then $S_{\hat{z}}$ meets $V_{i} \subset U_{i}^{\prime}$ and, since the pair $\left(U_{i}, U_{i}^{\prime}\right)$ is regular, there is a uniquely determined point $w^{\prime} \in S_{\hat{z}}$ which is at the minimal distance from $S_{\hat{z}}$ to $w$, moreover $w^{\prime} \in V_{i}$. We then define a local map $\bar{f}: V_{i}^{\prime} \rightarrow V_{i} \subset M$ by $\bar{f}(w)=w^{\prime}$. It follows from properties (P1) and (P2) that the above definition does not depend on the choice of $i=1, \ldots, m$. Indeed, if $w \in V_{i}^{\prime} \cap V_{j}^{\prime}$ then the points $w^{\prime}$ constructed using $U_{i}$ or $U_{j}$ belong to $V_{i} \cap V_{j}$ and are necessarily the same. This fact implies that the map $\bar{f}$ is globally defined in the entire manifold $M$. 
Since $f$ preserves leaves and is transversely holomorphic, it induces local holomorphic transformations $\hat{f}_{i}$ between open subsets of $\Delta_{i}=\phi_{i}\left(U_{i}\right) \subset \mathbb{C}^{n}$. Notice that, by construction, $\bar{f}$ sends leaves into leaves and that the local transverse part of $\bar{f}$ in $U_{i}$ is just $\hat{f}_{i}$.

Let us prove that $\bar{f}$ is a smooth diffeomorphism. We first notice that, if $w^{\prime}=\bar{f}(w)$ with $w \in V_{i}^{\prime}$, then the geodesic joining $w$ and $w^{\prime}$ and giving the minimal distance between $w$ and the slice $S_{z^{\prime}}$, where $z^{\prime}=\phi_{i}\left(w^{\prime}\right)$, meets $S_{z^{\prime}}$ orthogonally. By applying (10), we have

$$
\bar{f}(w)=w^{\prime}=p \circ \psi_{i}^{-1}\left(\phi_{i}(f(w)), w\right),
$$

Using the local transverse part $\hat{f}_{i}$ of $f$ the above equality can be written as

$$
\bar{f}(w)=w^{\prime}=p\left(\psi_{i}^{-1}\left(\hat{f}_{i}\left(\phi_{i}(w)\right), w\right)\right) .
$$

This equality proves that $\bar{f}$ is smooth and that $\bar{f}$ is entirely determined by the set of local transformations $\left\{\hat{f}_{i}\right\}$.

Restricted to $V_{i}^{\prime}$ the map $\bar{f}$ has an inverse that can be described as follows. Given $w^{\prime}=$ $\bar{f}(w) \in V_{i}$ we put $z^{\prime}=\phi_{i}\left(w^{\prime}\right)$ and $z=\phi_{i}(w)=\hat{f}_{i}^{-1}\left(z^{\prime}\right)$. As pointed out before, $\exp \left(N_{w^{\prime}} \mathcal{F} \cap\right.$ $W_{i}$ ) meets the slice $S_{z}$ and the intersection is precisely the point $w \in S_{z}$. In fact, there is a unique vector $v=v\left(w^{\prime}\right) \in N_{w^{\prime}} \mathcal{F} \cap W_{i}$ such that $w=\exp \left(w^{\prime}, v\right)$. Therefore we can write

$$
w=\bar{f}^{-1}\left(w^{\prime}\right)=\exp \left(w^{\prime}, v\right)=\pi_{2} \circ \psi_{i}\left(w^{\prime}, v\left(w^{\prime}\right)\right)
$$

and, since $\psi_{i}$ is a diffeomorphism, the vector $v=v\left(w^{\prime}\right)$ depends smoothly on $w^{\prime}$. Indeed, if we set $T=\exp \left(N_{w^{\prime}} \mathcal{F} \cap W_{i}\right) \cap V_{i}$ then $\left.\phi_{i}\right|_{T}$ maps $T$ isomorphically onto $\phi_{i}\left(V_{i}\right) \subset \Delta_{i}$ and we have

$$
\left(w^{\prime}, v\left(w^{\prime}\right)\right)=\psi_{i}^{-1}\left(z,\left(\left.\phi_{i}\right|_{T}\right)^{-1}(z)\right) .
$$

We deduce in particular that $\bar{f}$ is locally injective and that its (local) inverse is smooth. This also proves that the map $\bar{f}$ is open and, as the manifold $M$ is compact, $\bar{f}$ is necessarily surjective.

We remark that formula (13) also shows that $\bar{f}$ is entirely determined by the set of local transformations $\left\{\hat{f}_{i}\right\}$. That formula also shows that $\bar{f}$ depends continuously on $f \in \mathcal{U}$. Since the transverse part of $\bar{f}$ is just $\hat{f}$, the map $\bar{f}$ is transversely holomorphic. It remains to prove that $\bar{f}$ is globally injective and therefore an element of $\operatorname{Aut}(\mathcal{F}) \subset \mathcal{D}$.

Assume that $w^{\prime}=\bar{f}\left(w_{1}\right)=\bar{f}\left(w_{2}\right)$ and choose $i, j \in\{1, \ldots, m\}$ with $w_{1} \in V_{i}^{\prime}$ and $w_{2} \in V_{j}^{\prime}$. Then $V_{i} \cap V_{j} \neq \emptyset$ and therefore $V_{i} \cap V_{j} \subset U_{i}^{\prime}$. We deduce that $w_{1}, w_{2}$ belong to the same slice $S_{z}$ of $U_{i}$ where $z=\hat{f}_{i}^{-1}\left(\phi_{i}\left(w^{\prime}\right)\right)$. Now $w_{1}, w_{2}$ are in the intersection of $S_{z}$ with the submanifold $\exp \left(N_{w^{\prime}} \mathcal{F} \cap W_{i}\right) \cap V_{i}$. But this intersection reduces to a unique point, which shows that $w_{1}=w_{2}$.

Summarizing the above considerations, we see that the correspondence $f \mapsto \bar{f}=\Phi(f)$ given by (13) defines a continuous map $\Phi: \mathcal{V} \rightarrow \mathcal{D}$ with image $\tilde{\Sigma}=\Phi(\mathcal{V})$ contained in $\operatorname{Aut}(\mathcal{F})$. By construction, the diffeomorphism $f_{L}:=f \circ \bar{f}^{-1}$ is an element of $\mathcal{V}_{\mathcal{L}}$ and $\Phi$ maps $\mathcal{V}_{\mathcal{L}}$ to the identity. It is clear from the construction that each element $f \in \mathcal{V}$ decomposes in a unique way as the composition

$$
f=f_{L} \circ \bar{f}
$$

with $\bar{f}=\Phi(f) \in \tilde{\Sigma}$ and $f_{L} \in \mathcal{V}_{\mathcal{L}}$ and that $\bar{f}$ and $f_{L}$ depend continuously on $f$, with respect to the Fréchet topology. This tells us that the composition map $\Psi: \mathcal{V}_{\mathcal{L}} \times \tilde{\Sigma} \rightarrow \mathcal{V}$ defined in (7) has a continuous inverse. Therefore, by shrinking the set $\mathcal{V}_{\mathcal{L}}$ if it is necessary, the map $\Phi$ is a homeomorphism and the diagram (8) is commutative. 
Remark 4.7 Notice that $\tilde{\Sigma}$ is not necessarily closed under composition or taking inverses, even for elements close to the identity.

Remark 4.8 The construction carried out in the proof of the above proposition also provides a commutative diagram of continuous maps

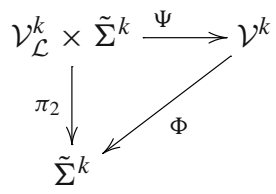

where $\mathcal{V}^{k}$ and $\mathcal{V}_{\mathcal{L}}^{k}$ are open neighborhoods of the identity in the $\operatorname{Sobolev}$ completions $\operatorname{Aut}^{k}(\mathcal{F})$ and $\mathcal{D}_{\mathcal{L}}^{k}$ respectively, the maps $\Phi$ and $\Psi$ are defined in the same way and $\Psi$ is a homeomorphism.

Remark 4.9 Assume for a moment that $\mathcal{F}$ is a smooth foliation without any particular geometric transverse structure. We notice that the correspondence $f \mapsto \bar{f}=\Phi(f)$ carried out in the above proposition is still well defined for any $f \in \mathcal{D}_{\mathcal{F}}$ close enough to the identity, since the transverse holomorphy of the diffeomorphisms plays no role in the construction of $\Phi$. In fact Proposition 4.6 can be stated by substituting the group $\operatorname{Aut}(\mathcal{F})$ for any closed subgroup $\mathcal{K}$ of $\mathcal{D}$ fulfilling $\mathcal{D}_{\mathcal{L}} \subset \mathcal{K} \subset \mathcal{D}_{\mathcal{F}}$. The proof of such a statement is the same as the proof of Proposition 4.6 without minor changes.

The following proposition states that, after shrinking it, if necessary, the set $\tilde{\Sigma}$ is naturally identified with an open neighborhood of the unity in the simply-connected Lie group $G$ associated to the Lie algebra $\mathcal{G}$. More precisely, if we identify $\mathcal{G}$ with the space $\mathfrak{X}_{N, b}$ of transversely holomorphic basic vector fields on $M$ that are orthogonal to $\mathcal{F}$, then we have:

Proposition 4.10 There is a neighborhood $\Sigma$ of the unity in $G$ and a homeomorphism $\sigma: \Sigma \rightarrow \tilde{\Sigma}$ such that the composition

$$
\hat{\Phi}:=\sigma^{-1} \circ \Phi: \mathcal{V} \rightarrow \Sigma
$$

preserves multiplication and inverses, i.e. $\hat{\Phi}$ is a morphism of local topological groups. Moreover, the composition

$$
U \subset \mathcal{G} \equiv \mathfrak{X}_{N, b} \rightarrow \Sigma,
$$

defined as $[\xi] \rightarrow \hat{\Phi} \circ \varphi_{1}^{\xi}$ for vector fields $\xi$ in $\mathfrak{X}_{N, b}$ close to zero, is the restriction to a neighborhood of zero of the natural exponential map $\mathcal{G} \rightarrow G$.

Here, $\varphi_{t}^{\xi}$ stands for the one-parameter group associated to $\xi$.

Proof We assume, as in the proof of the above proposition, that a finite family of adapted and cubic local charts $\left\{\left(U_{i}, \varphi_{i}\right)\right\}_{i=1, \ldots, m}$, and a family of 4-tuples $\left\{\left(U_{i}, U_{i}^{\prime}, V_{i}, V_{i}^{\prime}\right)\right\}_{i=1, \ldots, m}$ fulfilling conditions (C1), (C2) and (C3), have been fixed. As before we set $\phi=\pi_{2} \circ \varphi$ and we denote by $\Delta_{i}$ the polydisc $\phi_{i}\left(U_{i}\right)$ of $\mathbb{C}^{q}$. We also set $D_{i}=\phi_{i}\left(V_{i}\right)$ and $D_{i}^{\prime}=\phi_{i}\left(V_{i}^{\prime}\right)$. Let $B_{i}$ be the Banach space of continuous maps $f: \bar{D}_{i} \rightarrow \mathbb{C}^{q}$ that are holomorphic on $D_{i}$ with the norm

$$
|f|_{i}=\max _{z \in \bar{D}_{i}}|f(z)|
$$


Then the space $\mathcal{B}=\oplus_{i} B_{i}$ with the norm

$$
\|F\|=\left\|\left(f_{1}, \ldots, f_{m}\right)\right\|=\sum_{i}\left|f_{i}\right|
$$

is also a Banach space. Notice that $I=\left(\operatorname{Id}_{1}, \ldots, \mathrm{Id}_{m}\right)$, where $\operatorname{Id}_{i}$ denotes the identity map of $D_{i}$, is an element of $\mathcal{B}$.

We claim that there is an open neighborhood $\mathcal{I}_{\epsilon}=\{F \in \mathcal{B} \mid\|F-I\|<\epsilon\}$ of $I$ in $\mathcal{B}$ such that each $F=\left(f_{1}, \ldots, f_{m}\right) \in \mathcal{I}_{\epsilon}$ fulfills: (i) $f_{i}\left(\bar{D}_{i}^{\prime}\right) \subset D_{i}$ and (ii) $\left.f_{i}\right|_{D_{i}^{\prime}}$ is a biholomorphism onto its image for $i=1, \ldots, m$. Indeed, we know from Cauchy's inequalities that, for a given $\epsilon^{\prime}>0$ there is $\epsilon>0$ such that $\|F-I\|<\epsilon$ implies that $\left\|f_{i}-\operatorname{Id}_{i}\right\|_{C^{1}}<\epsilon^{\prime}$, where $\|\cdot\|_{C^{1}}$ stands for the $C^{1}$-norm on the polydisc $D_{i}^{\prime}$. Using the mean value theorem we see that, for $z, z^{\prime} \in D_{i}^{\prime}$, one has

$$
\left|f_{i}(z)-f_{i}\left(z^{\prime}\right)\right| \geq c\left|z-z^{\prime}\right|
$$

for a suitable constant $c>0$. Inequality (15) implies that the restriction of $f_{i}$ to the polydisc $D_{i}^{\prime}$ is injective and it is known that a one-to-one holomorphic map is necessarily a biholomorphism onto its image. Therefore, there is $\epsilon<0$ such that condition (ii) holds for $F \in \mathcal{I}_{\epsilon}$ and condition (i) is also fulfilled if $\epsilon$ is small enough. We assume that such an $\epsilon$, and therefore the open subset $\mathcal{I}_{\epsilon}$ of $\mathcal{B}$, have been fixed.

We consider now the holonomy pseudogroup $\mathcal{H}$ of the transversely holomorphic foliation $\mathcal{F}$. As a total transversal of $\mathcal{F}$ we choose the union $T=\cup T_{i}$ of disjoint submanifolds $T_{i}$ of $X$ of dimension $2 q$ and transverse to $\mathcal{F}$ with the property that $T_{i} \subset V_{i}^{\prime}$ and $\phi_{i}\left(T_{i}\right)=D_{i}^{\prime}$. Notice that $T$ is naturally endowed with a complex structure. We identify $T$, through the maps $\phi_{i}$, with the disjoint union of open polydiscs $T \equiv \amalg D_{i}^{\prime}$. The holonomy of the foliation $\mathcal{F}$ induces local transformations between open subsets of the total transversal $T$. They are holomorphic and $\mathcal{H}$ is the pseudogroup generated by these holonomy transformations. Since the manifold $X$ is compact, $\mathcal{H}$ is a compactly generated pseudogroup. This means that $\mathcal{H}$ is generated by a finite number of transformations of the pseudogroup, $h_{\mu}: W_{\mu} \rightarrow T$ for $\mu=1, \ldots, \ell$, with the following properties: $W_{\mu}$ is a relatively compact subset of some $D_{i}^{\prime}$ and $h_{\mu}$ has an extension to an open neighborhood of $\bar{W}_{\mu}$ in $D_{i}^{\prime}$ such that $h_{\mu}\left(\bar{W}_{\mu}\right) \subset D_{j}^{\prime}$ for some $1 \leq j \leq m$ (cf. [9]).

We define $\mathcal{S}$ as the subset of $\mathcal{I}_{\epsilon}$ of those $F=\left(f_{1}, \ldots, f_{m}\right)$ fulfilling

$$
f_{j}^{-1} \circ h_{\mu} \circ f_{i}=h_{\mu}
$$

in the common domain of definition. Clearly, $\mathcal{S}$ is closed in $\mathcal{I}_{\epsilon}$. We notice that each element $F=\left(f_{1}, \ldots, f_{m}\right) \in \mathcal{S}$ induces a well-defined diffeomorphism $\sigma(F)$ belonging to $\tilde{\Sigma}$. This correspondence $\sigma: \mathcal{S} \rightarrow \tilde{\Sigma}$ can be constructed as follows. Formula (13), where we replace $\hat{f}_{i}$ by the corresponding component $f_{i}$ of $F$, determines a (local) foliation preserving diffeomorphism $\bar{f}_{i}: V_{i}^{\prime} \rightarrow V_{i}$ whose transverse part is just $f_{i}$. The identities (16) guarantee that formula (13) applied to the family $\left\{f_{i}\right\}$ provides a well defined diffeomorphism $f=\sigma(F)$ of $M$ belonging to $\tilde{\Sigma}$. Clearly $\sigma$ maps $\mathcal{S}$ homeomorphically onto its image $\tilde{\Sigma}^{\prime}=\sigma(\mathcal{S})$. The fact that $f=\sigma(F)$ is a diffeomorphism globally defined over $M$ implies in particular that each one of the maps $f_{i}$ extends holomorphically to the polydisc $\Delta_{i}=\phi_{i}\left(U_{i}\right)$ and maps $\phi_{i}\left(U_{i}^{\prime}\right)$ biholomorphically onto an open subset of $\Delta_{i}$. From that fact one deduces easily that $\mathcal{S}$ is a local topological group (for the general properties of local topological groups we refer to [26]). Moreover, if we set $\mathcal{V}^{\prime}=\Phi^{-1}\left(\tilde{\Sigma}^{\prime}\right)$ then the composition

$$
\hat{\Phi}:=\sigma^{-1} \circ \Phi: \mathcal{V}^{\prime} \rightarrow \mathcal{S}
$$


can be seen as the map which associates its transverse part $\hat{f}$ to each diffeomorphism $f$ in $\operatorname{Aut}(\mathcal{F})$ which is close enough to the identity. It follows in particular that the map $\hat{\Phi}$ preserves multiplication and inverses. It is therefore a morphism of local groups.

The end of the proof will be based on the following fact.

Lemma 4.11 The local group $\mathcal{S}$ is locally compact.

Proof Let us fix $0<\epsilon^{\prime}<\epsilon$. It is sufficient to prove that the neighborhood $\mathcal{S}_{\epsilon^{\prime}}$ of $I$ defined by $\|F-I\| \leq \epsilon^{\prime}$ is compact. Let $\left\{F_{k}=\left(f_{k, 1}, \ldots, f_{k, m}\right)\right\}$ be a sequence of elements of $\mathcal{S}_{\epsilon^{\prime}}$. As noticed before, we can think of each $f_{k, i}$ as a holomorphic transformation defined on $\Delta_{i}$ that sends $\phi_{i}\left(U_{i}^{\prime}\right)$ into $\Delta_{i}$. By taking $\epsilon^{\prime}$ small enough, we can also assume that $f_{k, i}\left(\phi_{i}\left(U_{i}^{\prime}\right)\right)$ contains $D_{i}$. It follows from Montel's theorem that there is a subsequence $\left\{F_{k_{j}}\right\}$ of $\left\{F_{k}\right\}$ such that each $f_{k_{j}, i}$ converges uniformly to a holomorphic map $\tilde{f}_{i}$. Since $f_{k, i}$ are invertible, we can suppose that the sequences $\left\{f_{k_{j}}^{-1}\right\}$, that are defined on $D_{i}$, also converge to a limit which necessarily is $\tilde{f}_{i}^{-1}$. This proves that $\left\{F_{k_{j}}\right\}$ has a limit that belongs to $\mathcal{S}_{\epsilon^{\prime}}$.

End of proof of Proposition 4.10 Now we are in position to apply Theorem A in [2] to the local group $\mathcal{S}$. Although the result by Bochner and Montgomery is stated in the context of topological groups, the arguments used there are purely local and therefore they still remain valid for locally compact local groups of transformations. It implies in particular that $\mathcal{S}$ is a local Lie group, i.e. $\mathcal{S}$ is isomorphic to a neighborhood of the identity of a certain Lie group.

Finally, we claim that this Lie group is just the 1-connected Lie group $G$ associated to the Lie algebra $\mathcal{G}$. Indeed, each element $\xi$ of $\mathfrak{X}_{N, b} \equiv \mathcal{G}$ induces a one parameter group $\varphi_{t}^{\xi}$ that is sent by $\hat{\Phi}$ (and for small $t$ ) into a local one parameter subgroup of $\mathcal{S}$ which does not reduce to the identity unless $\xi=0$. Conversely, each local one parameter subgroup of $\mathcal{S}$ is obtained in this way. Hence $\mathcal{S}$ is naturally identified to a neighborhood $\Sigma$ of the unity in $G$. The last assertion of the proposition is clear from the above discussion.

Remark 4.12 The map $\sigma$ constructed in the above proposition also identifies $\Sigma$ with the set $\tilde{\Sigma}^{k}=\Phi\left(\mathcal{V}^{k}\right)$ considered in Remark 4.8

Remark 4.13 The following criterium is useful to assure that certain maps between Fréchet manifolds are smooth (cf. [10]). Let $V$ and $V^{\prime}$ be fibre bundles over a compact manifold $X$, eventually with boundary, and let $\Gamma(V)$ and $\Gamma\left(V^{\prime}\right)$ denote the Fréchet manifolds of the smooth sections of $V$ and $V^{\prime}$ respectively. Assume that there is an open subset $U \subset V$ meeting all the fibres of $V$ and a fibrewise smooth map $F: U \rightarrow V^{\prime}$ projecting onto the identity of $X$. Let $\tilde{U}$ be the subset of $\Gamma(V)$ of those sections $s$ having image in $U$. Then the map $\chi: \tilde{U} \subset \Gamma(V) \rightarrow \Gamma\left(V^{\prime}\right)$ given by $s \mapsto \chi(s)=F \circ s$ is a smooth map of Fréchet manifolds. Moreover, assume that $\mathcal{E}$ and $\mathcal{E}^{\prime}$ are Fréchet submanifolds of $\Gamma(V)$ and $\Gamma\left(V^{\prime}\right)$ respectively with $\mathcal{E} \subset \tilde{U}$ and such that $\chi(\mathcal{E}) \subset \mathcal{E}^{\prime}$, then the restricted map $\chi: \mathcal{E} \rightarrow \mathcal{E}^{\prime}$ is also smooth.

Combining the above two propositions we obtain the commutative diagram

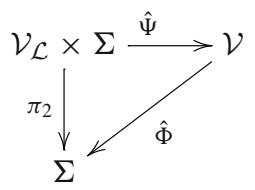

As a corollary we deduce the following 
Proposition 4.14 With the induced topology, $\operatorname{Aut}(\mathcal{F})$ is a closed strong ILH-Lie subgroup of $\mathcal{D}$ with Lie algebra $\mathfrak{a u t}(\mathcal{F})$.

Proof Notice first that $\operatorname{Aut}(\mathcal{F})$ is a closed subgroup of $\mathcal{D}$. Using the above commutative diagram as well as Remarks 4.8 and 4.12 , and arguing as in the proof of Proposition 2.7, we see that $\operatorname{Aut}^{\prime k}(\mathcal{F})$ coincides with $\operatorname{Aut}_{0}^{k}(\mathcal{F})$ (the connected component of $\operatorname{Aut}^{k}(\mathcal{F})$ containing the identity). This implies that $\operatorname{Aut}^{\prime}(\mathcal{F})$ is the connected component of the identity of $\operatorname{Aut}(\mathcal{F})$ concluding the proof.

End of proof of Theorem 4.3 We first prove that the map $\hat{\Phi}$ in diagram (17) is smooth. Let $\left\{\left(U_{i}, \varphi_{i}\right)\right\}_{i=1, \ldots, m}$ be a finite family of adapted and cubic local charts of $M$ and let $\left\{\left(U_{i}, U_{i}^{\prime}\right)\right\}$ be a family of regular pairs with the properties that $\left\{U_{i}^{\prime}\right\}$ is an open cover of $M$ and $\phi_{i}\left(U_{i}\right)=D_{i}$ and $\phi_{i}\left(U_{i}^{\prime}\right)=D_{i}^{\prime}$ are open polydiscs. We choose a family $\left\{T_{i}\right\}$ of disjoint transversals $T_{i} \subset U_{i}$ of $\mathcal{F}$ such that $\phi_{i}\left(T_{i}\right)=D_{i}$. We set $T_{i}^{\prime}=T_{i} \cap \bar{U}_{i}^{\prime}$ and we identify $T=\amalg T_{i}^{\prime}$, through the maps $\phi_{i}$, with the disjoint union of closed polydiscs $T=U \bar{D}_{i}^{\prime}$. The manifolds $V=\amalg\left(T_{i}^{\prime} \times U_{i}\right)$ and $W=\amalg\left(T_{i}^{\prime} \times D_{i}\right)$ are fibre bundles over $T$ taking as projection the natural projections onto the first factor. The map $F: V \rightarrow W$ given by $F\left(z_{i}, w_{i}\right)=\left(z_{i}, \phi_{i}\left(w_{i}\right)\right)$ is fibrewise and smooth, and it follows from the criterium stated in Remark 4.13 that the map $\chi: \Gamma(V) \rightarrow \Gamma(W)$, given by $\chi(s)=F \circ s$, is smooth. Notice that diffeomorphisms of M close to the identity can be thought of sections of the fibre bundle $\pi_{1}: M \times M \rightarrow M$ with image close to the diagonal. Moreover, for a small enough neighborhood $\mathcal{W}$ of the identity in $\mathcal{D}$, the map

$$
\begin{array}{ccc}
\tau: \mathcal{W} & \longrightarrow & \Gamma(V) \\
f & \mapsto\left\{z_{i} \mapsto\left(z_{i}, f\left(z_{i}\right)\right)\right\}
\end{array}
$$

is well defined and smooth. If $f \in \mathcal{W}$ belongs to $\operatorname{Aut}(\mathcal{F})$ the family $\left\{z_{i}, \phi_{i}\left(f\left(z_{i}\right)\right)\right\}=$ $\chi(\tau(f))$ is an element of the local group $\mathcal{S} \equiv \Sigma$ constructed in the proof of Proposition 4.10. Therefore the map $\hat{\Phi}: \mathcal{V} \rightarrow \Sigma$ can be written as the restriction to $\mathcal{V} \subset \operatorname{Aut}(\mathcal{F})$ of the composition $\chi \circ \tau$, which proves that it is smooth.

Let us consider now the map $\sigma: \Sigma \equiv \mathcal{S} \rightarrow \tilde{\Sigma} \subset \operatorname{Aut}(\mathcal{F})$. A reasoning similar to the previous one, using now the local expression of the correspondence $s=\left\{f_{i}\right\} \in \mathcal{S} \mapsto \bar{f} \in \tilde{\Sigma}$ given by the equality (13), shows that $\sigma$ is smooth and therefore that $\Psi: \mathcal{V}_{\mathcal{L}} \times \Sigma \rightarrow \mathcal{V}$, which is given by the multiplication $\hat{\Psi}(f, s)=f \circ \sigma(s)$, is also smooth. Its inverse $\hat{\Psi}^{-1}$ is just the correspondence $f \mapsto\left(f_{L}, f_{N}\right)$, where $f_{N}=\hat{\Phi}(f)$ and $f_{L}=f \circ f_{N}^{-1}$, which proves that $\hat{\Psi}$ is a diffeomorphism.

Therefore, the map $\hat{\Psi}: \mathcal{V}_{\mathcal{L}} \times \Sigma \rightarrow \mathcal{V}$ can be regarded as a local chart of $\operatorname{Aut}(\mathcal{F})$ and the map $\hat{\Phi}: \mathcal{V} \rightarrow \Sigma$ as a submersion defining the foliation $\mathcal{F}_{\mathcal{D}}$ in a neighborhood of the identity.

Let $f \in \operatorname{Aut}_{0}(\mathcal{F})$ be given. As each connected topological group, $\operatorname{Aut}_{0}(\mathcal{F})$ is generated, as a group, by a given neighborhood of the identity, in particular by $\mathcal{V} \cong \mathcal{V}_{\mathcal{L}} \times \tilde{\Sigma}$. It follows, using that $\mathcal{D}_{\mathcal{L}}$ is a normal subgroup of $\operatorname{Aut}_{0}(\mathcal{F})$, that we can write

$$
f=f_{L} \circ f_{N}=f_{L} \circ \exp \left(v_{1}\right) \circ \cdots \circ \exp \left(v_{k}\right)
$$

where $f_{L} \in \mathcal{D}_{\mathcal{L}}$ and $v_{i} \in \mathfrak{X}_{N, b}$ are vector fields with the property that, if we denote $\hat{v}_{i}=\vartheta\left(v_{i}\right) \in \mathcal{G}$, where $\vartheta: \mathfrak{X}_{N, b} \rightarrow \mathcal{G}$ is the natural identification, then $\exp \left(\hat{v}_{i}\right) \in \Sigma$. We denote by $\hat{f}_{N}$ the element of $G$ given by

$$
\hat{f}_{N}=\exp \left(\hat{v}_{1}\right) \circ \cdots \circ \exp \left(\hat{v}_{k}\right)
$$


Now, proceeding as before we can prove that there exist neighborhoods $\mathcal{V}_{f} \subset \operatorname{Aut}(\mathcal{F})$, $\mathcal{V}_{\mathcal{L}, f} \subset \mathcal{D}_{\mathcal{L}}$ and $\Sigma_{f} \subset G$, of $f, f_{L}$ and $\hat{f}_{N}$ respectively, and a commutative diagram

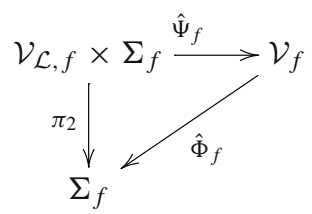

where $\hat{\Psi}_{f}$ is a diffeomorphism providing a local chart of $\operatorname{Aut}(\mathcal{F})$ and $\hat{\Phi_{f}}$ is a smooth submersion defining $\mathcal{F}_{\mathcal{D}}$ in $\mathcal{V}_{f}$. Finally, it also follows from the above discussion that, if $\mathcal{V}_{f_{i}}$ and $\mathcal{V}_{f_{j}}$ have non-empty intersection, then there are locally constant $G$-valued functions $\gamma_{i j}$ fulfilling

$$
\hat{\Phi}_{f_{i}}=L_{\gamma_{i j}} \circ \hat{\Phi}_{f_{j}}
$$

This ends the proof of the theorem

Remark 4.15 The Kuranishi property can also be formulated in the setting of transversely holomorphic foliations. It follows from the versality theorem in [7] that each transversely holomorphic foliation on a compact manifold has the Kuranishi property.

\section{The automorphism group of a Riemannian foliation}

We suppose in this section that the foliation $\mathcal{F}$ on $M$ is Riemannian. This means that the local submersions $\phi_{i}$ defining the foliation take values in a Riemannian manifold $T$ and that the transformations $\gamma_{i j}$ fulfilling the cocycle condition (1) are local isometries of $T$. In an equivalent way, the foliation $\mathcal{F}$ is Riemannian if there is a Riemannian metric $g$ on $M$ which is bundle-like with respect to $\mathcal{F}$, that is a metric that in local adapted coordinates $(x, y)$ is written

$$
g=\sum g_{i j}(x, y) \omega^{i} \omega^{j}+\sum g_{a b}(y) d y^{a} d y^{b},
$$

where $\left\{\omega^{i}, d y^{a}\right\}$ is a local basis of 1 -forms such that $\omega^{i}$ vanish on the bundle of vectors orthogonal to $\mathcal{F}$.

We suppose that such a bundle-like metric $g$ has been fixed. Let $p$ and $q$ stand for the dimension and the codimension of $\mathcal{F}$ respectively.

In this section $\operatorname{Aut}(\mathcal{F})$ will denote the automorphism group of the Riemannian foliation $\mathcal{F}$, that is the group of elements of $\mathcal{D}_{\mathcal{F}}$ which preserve the transverse Riemannian metric, and by $\mathfrak{a u t}(\mathcal{F})$ the Lie algebra of vector fields whose flows are one-parameter subgroups of $\operatorname{Aut}(\mathcal{F})$. Also in this case, $\mathcal{D}_{\mathcal{L}}$ is a normal subgroup of $\operatorname{Aut}(\mathcal{F})$ and $\mathfrak{X}_{\mathcal{L}}$ is an ideal of $\mathfrak{a u t}(\mathcal{F})$. We denote by $\mathcal{G}=\mathfrak{a u t}(\mathcal{F}) / \mathfrak{X}_{\mathcal{L}}$ the quotient Lie algebra. The elements of $\mathcal{G}$ generate local isometries on $T$, therefore they are called basic Killing vector fields.

Proposition 5.1 The Lie algebra $\mathcal{G}$ of basic Killing vector fields has finite dimension.

The above proposition can be proved in the same way as the classical theorem of Myers and Steenrod (cf. [13,20]). Namely, let $\pi: P \rightarrow M$ be the $O(q)$-principal fibre bundle of transverse orthonormal frames of the Riemannian foliation $\mathcal{F}$. The total space $P$ is endowed with a foliation $\mathcal{F}_{P}$ of dimension $p$ that is projected by $\pi$ to $\mathcal{F}$. The foliation $\mathcal{F}_{P}$ is transversely parallelizable and the elements of $\operatorname{Aut}(\mathcal{F})$ are in one-to-one correspondence with the 
automorphisms of the principal fibre bundle which preserve the transverse parallelism and the Riemannian connection. The finiteness of $\mathcal{G}$ then follows from the general fact according to which the automorphism group of a parallelism is finite dimensional.

In particular we can consider the simply connected Lie group associated to the Lie algebra $\mathcal{G}$ that we denote $G$.

As we already mentioned Leslie proved in [16] that, in the case of a Riemannian foliation, the group $\mathcal{D}_{\mathcal{F}}$ is a Fréchet Lie group. Moreover, using the connection provided by the Riemannian metric, Omori's proof that $\mathcal{D}$ is a strong ILH-Lie group can be adapted to show the following

Proposition 5.2 Let $\mathcal{F}$ be a Riemannian foliation on a compact manifold $M$. Then $\mathcal{D}_{\mathcal{F}}$ is an ILH-Lie group with Lie algebra $\mathfrak{X}_{\mathcal{F}}$.

Remark 5.3 The structure of the group $\mathcal{D}_{\mathcal{F}}$ when $\mathcal{F}$ is a Riemannian foliation on a non necessarily compact manifold has been studied in [17].

Our main result in this section is the following theorem.

Theorem 5.4 Let $\mathcal{F}$ be a Riemannian foliation on a compact manifold $M$. The automorphism group $\operatorname{Aut}(\mathcal{F})$ of $\mathcal{F}$ is closed in $\mathcal{D}_{\mathcal{F}}$ and, with the induced topology, it is a strong ILH-Lie group with Lie algebra $\mathfrak{a u t}(\mathcal{F})$. Moreover, the left cosets of the subgroup $\mathcal{D}_{\mathcal{L}}$ define a Lie foliation $\mathcal{F}_{\mathcal{D}}$ on $\operatorname{Aut}(\mathcal{F})$, which is transversely modeled on the simply-connected complex Lie group $G$ associated to the Lie algebra $\mathcal{G}$.

Remark 5.5 The above theorem is weaker than Theorem 4.3 as we are not able to exhibit a supplementary to the Lie subalgebra $\mathfrak{a u t}(\mathcal{F})$ inside the Lie algebra $\mathfrak{X}$ of $\mathcal{D}$. Consequently we do not show that $\operatorname{Aut}(\mathcal{F})$ is a strong ILH-Lie subgroup of $\mathcal{D}$. Notice however that Theorem 5.4 implies in particular that the topology of $\operatorname{Aut}(\mathcal{F})$ is second countable and LPSAC.

The proof of the above result is parallel to that of Theorem 4.3, hence we just indicate the differences.

Proof (Sketch of proof) We recall first that the correspondence $f \mapsto \bar{f}=\Phi(f)$ given by Proposition 4.6 is well defined for each $f \in \mathcal{D}_{\mathcal{F}}$ close enough to the identity (cf. Remark 4.9). The proof of that proposition shows that there is a commutative diagram of continuous maps

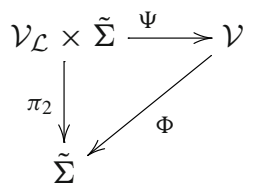

where $\mathcal{V}$ and $\mathcal{V}_{\mathcal{L}}$ are neighborhoods of the identity in $\operatorname{Aut}(\mathcal{F})$ and $\mathcal{D}_{\mathcal{F}}$ respectively, $\tilde{\Sigma}$ is the image of $\Phi$ and the map $\Psi$, that is given by multiplication, is a homeomorphism. As stated in Remark 4.8 the same proof also provides commutative diagrams of continuous maps between the Sobolev completions of the spaces in (18).

Proceeding as in Proposition 4.10, we consider a complete transversal $T$ to $\mathcal{F}$ which now is endowed with a Riemannian metric. The families of local isometries of $T$ that are close to the identity and that commute with the holonomy pseudogroup of $\mathcal{F}$ are the elements of a local group $\mathcal{S}$ that can be identified to $\tilde{\Sigma}$. We claim that $\mathcal{S}$ is locally compact. In the present setting this fact follows from the following observation that replaces Lemma 4.11. A local isometry is determined, on a connected domain, by its 1-jet at a given point, therefore $\mathcal{S}$ can 
be embedded in a space of 1 -jets over $T$, which is finite dimensional. Moreover, $\mathcal{S}$ is locally closed in this space of jets and therefore it is locally compact.

The arguments used by Salem in [27] can be applied here to show that $\mathcal{S}$ is in fact a local Lie group, more precisely each element of $\mathcal{S}$ is in the flow of a Killing vector field over $T$ that commutes with the holonomy pseudogroup of $\mathcal{F}$. These Killing vector fields form a Lie algebra naturally isomorphic to $\mathcal{G}$ and therefore $\tilde{\Sigma}$ is identified, through a suitable map $\sigma$, to a neighborhood $\Sigma$ of the neutral element of the simply connected Lie group $G$. Notice that isometries of a smooth Riemannian metric are necessarily of class $C^{\infty}$. Therefore, and as it happens in Remark 4.12, the map $\sigma$ also identifies $\Sigma$ with the set $\tilde{\Sigma}^{k}=\Phi\left(\mathcal{V}^{k}\right)$ for $k$ big enough.

The maps $\hat{\Psi}: \mathcal{V}_{\mathcal{L}} \times \Sigma \rightarrow \mathcal{V}$ and $\hat{\Psi}: \mathcal{V}_{\mathcal{L}}^{k} \times \Sigma \rightarrow \mathcal{V}^{k}$ can be regarded as local charts of $\operatorname{Aut}(\mathcal{F})$ and $\operatorname{Aut}^{k}(\mathcal{F})$ respectively. Moreover the map $\hat{\Phi}: \mathcal{V} \rightarrow \Sigma$ is a submersion defining the foliation $\mathcal{F}_{\mathcal{D}}$ in a neighborhood of the identity. In a similar way as in the proof of Theorem 4.3, one can construct atlases of adapted local charts for $\operatorname{Aut}(\mathcal{F})$ and $\operatorname{Aut}^{k}(\mathcal{F})$. In particular the groups $\operatorname{Aut}^{k}(\mathcal{F})$ turn out to be Hilbert manifolds showing that $\operatorname{Aut}(\mathcal{F})$ is an ILH-Lie group. This ends the proof.

Remark 5.6 In Appendix E of [20], E. Ghys raised the problem of developing a theory of deformations for Riemannian foliations and conjectured the existence of a versal deformation parametrized by a finite dimensional space. Up to now there are not general answers to that question. In particular, we do not know if Riemannian foliations on compact manifolds fulfill the corresponding Kuranishi property.

Funding Open Access Funding provided by Universitat Autonoma de Barcelona.

Open Access This article is licensed under a Creative Commons Attribution 4.0 International License, which permits use, sharing, adaptation, distribution and reproduction in any medium or format, as long as you give appropriate credit to the original author(s) and the source, provide a link to the Creative Commons licence, and indicate if changes were made. The images or other third party material in this article are included in the article's Creative Commons licence, unless indicated otherwise in a credit line to the material. If material is not included in the article's Creative Commons licence and your intended use is not permitted by statutory regulation or exceeds the permitted use, you will need to obtain permission directly from the copyright holder. To view a copy of this licence, visit http://creativecommons.org/licenses/by/4.0/.

\section{References}

1. Benhenda, M.: Circle diffeomorphisms: quasi-reducibility and commuting diffeomorphisms. Nonlinearity 25(7), 1981-1995 (2012)

2. Bochner, S., Montgomery, D.: Locally compact groups of differentiable transformations. Ann. Math. (2) 47, 639-653 (1946)

3. Donaldson, S.K.: An application of gauge theory to four dimensional topology. J. Differ. Geom. 18, 269-278 (1983)

4. Duchamp, T., Kalka, M.: Deformation theory for holomorphic foliations. J. Differ. Geom. 14(3), 317-337 (1979)

5. Ebin, D.G.: The manifold of Riemannian metrics. Global Analysis (Proc. Sympos. Pure Math., vol. XV, Berkeley, Calif., 1968), pp. 11-40, Amer. Math. Soc., Providence (1970)

6. Ebin, D.G., Marsden, J.: Groups of diffeomorphisms and the motion of an incompressible fluid. Ann. Math. (2) 92, 102-163 (1970)

7. Girbau, J., Haefliger, A., Sundararaman, D.: On deformations of transversely holomorphic foliations. J. Reine Angew. Math. 345, 122-147 (1983)

8. Gómez-Mont, X.: Transversal holomorphic structures. J. Differ. Geom. 15(2), 161-185 (1980)

9. Haefliger, A.: Pseudogroups of local isometries. Differential geometry (Santiago de Compostela, 1984), pp. 174-197, Res. Notes in Math., vol. 131. Pitman, Boston (1985) 
10. Hamilton, R.S.: The inverse function theorem of Nash and Moser. Bull. Am. Math. Soc. (N.S.) 7(1), 65-222 (1982)

11. Heitsch, J.L.: A cohomology for foliated manifolds. Comment. Math. Helv. 50, 197-218 (1975)

12. Herman, M.-R.: Sur la conjugaison différentiable des difféomorphismes du cercle à des rotations. Inst. Hautes Études Sci. Publ. Math. 49, 5-233 (1979)

13. Kobayashi, S.: Transformation Groups in Differential Geometry. Springer, Berlin (1995)

14. Kopell, N.: Commuting diffeomorphisms. In: Global Analysis, Proc. of Symp. in Pure Math., vol. XIV. AMS (1970)

15. Leslie, J.A.: On a differential structure for the group of diffeomorphisms. Topology 6, 263-271 (1967)

16. Leslie, J.A.: Two classes of classical subgroups of Diff(M). J. Differ. Geom. 5, 427-435 (1971)

17. Macias-Virgós, E., Sanmartín Carbón, E.: Manifolds of maps in Riemannian foliations. Geom. Dedicata 79(2), 143-156 (2000)

18. Meersseman, L.: Variétés CR polarisées et G-polarisées, partie I. Int. Math. Res. Not. IMRN 21, 59125973 (2014)

19. Meersseman, L., Nicolau, M.: Deformations and moduli of structures on manifolds: general existence theorem and application to the Sasakian case. Ann. Sc. Norm. Super. Pisa Cl. Sci. (5) 17(1), 19-63 (2017)

20. Molino, P.: Riemannian Foliations. Progress in Mathematics, vol. 73. Birkhäuser, Boston (1988)

21. Morrow, J., Kodaira, K.: Complex Manifolds. Holt, Rinehart and Winston, New York (1971)

22. Omori, H.: On the group of diffeomorphisms on a compact manifold. Global Analysis (Proc. Sympos. Pure Math., Vol. XV, Berkeley, Calif., 1968), pp. 167-183 Amer. Math. Soc., Providence (1970)

23. Omori, H.: Infinite dimensional Lie transformation groups. Lecture Notes in Mathematics, vol. 427. Springer, Berlin (1974)

24. Omori, H.: Infinite-dimensional Lie groups. Translations of Mathematical Monographs, vol. 158. American Mathematical Society, Providence (1997)

25. Palais, R.S.: Foundations of Global Non-linear Analysis. W. A. Benjamin Inc, New York (1968)

26. Pontryagin, L.S.: Topological Groups. Gordon and Breach Science Publishers Inc, New York (1966)

27. Salem, E.: Une généralisation du théorème de Myers-Steenrod aux pseudogroupes d'isométries. Ann. Inst. Fourier 38(2), 185-200 (1988)

28. Yoccoz, J.-C.: Conjugaison différentiable des difféomorphismes du cercle dont le nombre de rotation vérifie une condition diophantienne. Ann. Sci. École Norm. Sup. (4) 17, 333-359 (1984)

29. Yoccoz, J.-C.: Petits diviseurs en dimension 1. Asterisque, 231 (1995)

Publisher's Note Springer Nature remains neutral with regard to jurisdictional claims in published maps and institutional affiliations. 